\title{
Axions and the Galactic Angular Momentum Distribution
}

\author{
N. Banik and P. Sikivie ${ }^{1}$ \\ ${ }^{1}$ Department of Physics, University of Florida, Gainesville, FL 32611, USA
}

(Dated: July 10, 2013)

\begin{abstract}
We analyze the behavior of axion dark matter before it falls into a galactic gravitational potential well. The axions thermalize sufficiently fast by gravitational self-interactions that almost all go to their lowest energy state consistent with the total angular momentum acquired from tidal torquing. That state is a state of rigid rotation on the turnaround sphere. It predicts the occurrence and detailed properties of the caustic rings of dark matter for which observational evidence had been found earlier. We show that the vortices in the axion Bose-Einstein condensate (BEC) are attractive, unlike those in superfluid ${ }^{4} \mathrm{He}$ and dilute gases. We expect that a large fraction of the vortices in the axion BEC join into a single big vortex along the rotation axis of the galaxy. The resulting enhancement of caustic rings explains the typical size of the rises in the Milky Way rotation curve attributed to caustic rings. We show that baryons and ordinary cold dark matter particles are entrained by the axion BEC and acquire the same velocity distribution. The resulting baryonic angular momentum distribution gives a good qualitative fit to the distributions observed in dwarf galaxies. We give estimates of the minimum fraction of dark matter that is axions.
\end{abstract}

PACS numbers: 95.35.+d 


\section{INTRODUCTION}

One of the outstanding problems in science today is the identity of the dark matter of the universe [1]. The existence of dark matter is implied by a large number of observations, including the dynamics of galaxy clusters, the rotation curves of individual galaxies, the abundances of light elements, gravitational lensing, and the anisotropies of the cosmic microwave background radiation. The energy density fraction of the universe in dark matter is observed to be $26.7 \%$ [2]. The dark matter must be non-baryonic, cold and collisionless. Non-baryonic means that the dark matter is not made of ordinary atoms and molecules. Cold means that the primordial velocity dispersion of the dark matter particles is sufficiently small, less than about $10^{-8} c$ today, so that it may be set equal to zero as far as the formation of large scale structure and galactic halos is concerned. Collisionless means that the dark matter particles have, in first approximation, only gravitational interactions. Particles with the required properties are referred to as 'cold dark matter' (CDM). The leading CDM candidates are weakly interacting massive particles (WIMPs) with mass in the $100 \mathrm{GeV}$ range, axions with mass in the $10^{-5} \mathrm{eV}$ range, and sterile neutrinos with mass in the keV range. To try and tell these candidates apart on the basis of observation is a tantalizing quest.

Recently it has been argued that the dark matter is axions [3 -5] because this assumption provides a natural explanation and detailed account of the existence and properties of caustic rings of dark matter in galactic halos. Axions are different from the other cold dark matter candidates, such as WIMPs and sterile neutrinos, because axions form a Bose-Einstein condensate (BEC). They do so as a result of their gravitational self-interactions. On time scales long compared to their thermalization time scale $\tau$, almost all axions go to the lowest energy state available to them. The other dark matter candidates do not do this. The (re)thermalization of the axion BEC is sufficiently fast that axions that are about to fall into a galactic gravitational potential well go to their lowest energy available state consistent with the total angular momentum they acquired from nearby protogalaxies through tidal torquing [5]. That state is a state of net overall rotation. In contrast, ordinary cold dark matter falls into galactic gravitational potential wells with an irrotational velocity field [6]. The inner caustics are different in the two cases. In the case of net overall rotation, the inner caustics are rings [7] whose cross-section is a section of the elliptic umbilic $D_{-4}$ catastrophe [8], called caustic rings for short. If the velocity field of the infalling particles is irrotational, the inner caustics have a 'tent-like' structure which is described in detail in ref. [6] and which is quite distinct from caustic rings. Evidence was found for caustic rings. A summary of

the evidence is given in ref. [9]. Furthermore, it was shown in ref. [4] that the assumption that the dark matter is axions explains not only the existence of caustic rings but also their detailed properties, in particular the pattern of caustic ring radii and their overall size.

The main purpose of the present paper is to give a more detailed description of the behavior of axion dark matter before it falls into the gravitational potential well of a galaxy. In particular we want to investigate the appearance and evolution of vortices in the rotating axion BEC, and ask whether they have implications for observation. We obtain two results that may be somewhat surprising. The first is that, unlike the vortices in superfluid ${ }^{4} \mathrm{He}$ and in BECs of dilute gases, the vortices in axion BEC attract each other. The reason for the difference in behavior is that atoms have short range repulsive interactions whereas axions do not. The vortices in the axion BEC join each other producing vortices of ever increasing size. When two vortices join, their radii (not their cross-sectional areas) are added. We 
expect a huge vortex to form along the rotation axis of the galaxy as the outcome of the joining of numerous smaller vortices. We call it the 'big vortex'. The presence of a big vortex implies that the infall is not isotropic as has been assumed in the past [7-10]. The axions fall in preferentially along the equatorial plane. Caustic rings are enhanced as a result because the density of the flows that produce the caustic rings is larger. We propose this as explanation of the fact that the rises in the Milky Way rotation curve attributed to caustic rings [11] are typically a factor five larger than predicted assuming that the infall is isotropic, a puzzle for which no compelling explanation had been given in the past.

The second perhaps surprising finding is that baryons are entrained by the axion BEC and acquire the same velocity distribution as the axion BEC. The underlying reason for this is that the interactions through which axions thermalize are gravitational and gravity is universal. The condition for the baryons to acquire net overall rotation by thermal contact with the axion $\mathrm{BEC}$ is the same as the condition for the axions to acquire net overall rotation by thermalizing among themselves. When baryons and axions are in thermal equilibrium, their velocity fields are the same since otherwise entropy can be generated by energy-momentum exchanging interactions between them. We expect that a big vortex forms in the baryon fluid as well, although one of lesser size than the big vortex in the axion fluid. The resulting angular momentum distribution of baryons agrees qualitatively with that observed by van den Bosch et al. in dwarf galaxies [12]. In contrast, if the dark matter is WIMPs or sterile neutrinos, the predicted angular momentum distribution of baryons in galaxies differs markedly from the observed distribution, a discrepancy known as the 'galactic angular momentum problem' [13, 14].

We consider the possibility that the dark matter is a mixture of axions and another form of cold dark matter. For the purposes of our discussion WIMPs and sterile neutrinos behave in the same way. So we call the other form of cold dark matter WIMPs for the sake of brevity. There is a minimum fraction of dark matter that must be axions for the axions to rethermalize by gravitational interactions before they fall into galactic gravitational potential wells. That fraction is of order $3 \%$. If the axion fraction of dark matter is larger than of order $3 \%$, the axions acquire net overall rotation through their thermalizing gravitational self-interactions and entrain the baryons and WIMPs with them. The baryons and WIMPs acquire the same velocity distribution as the axions before falling onto galactic halos. The WIMPs therefore produce the same caustic rings, and at the same locations, as the axions. However, to account for the typical size of the rises in the Milky Way rotation curve attributed to caustic rings, we find that the fraction of dark matter in axions must be of order $37 \%$ or more.

To investigate the issues of interest here we generalize the statistical mechanics of many body systems in thermal equilibrium to the case when the system is rotating and total angular momentum is conserved. When total angular momentum is conserved, a system of identical particles in thermal equilibrium is characterized by an angular frequency $\omega$ in addition to its temperature $T$ and its chemical potential $\mu$. Broadly speaking, the state of thermal equilibrium of such systems is one of rigid rotation with angular frequency $\omega$. Incidentally, we find that there is no satisfactory generalization of the isothermal sphere model of galactic halos with $\omega \neq 0$. This is a serious flaw of that model since galactic halos acquire angular momentum from tidal torquing. For a Bose-Einstein condensate, we derive the state that most particles condense into when $\omega \neq 0$. In superfluid ${ }^{4} \mathrm{He}$ this state is one of rigid rotation except for a regular array of vortices embedded in the fluid. For a BEC of collisionless particles contained in a cylindrical volume the state is one of quasi-rigid rotation 
with all the particles as far removed from the axis of rotation as allowed by the Heisenberg uncertainty principle. For an axion BEC about to fall into a galactic gravitational well the state is one in which each spherical shell rotates rigidly but the rotational frequency varies with the shell's radius $r$ as $r^{-2}$. (The motion is similar to that of water draining through a hole in a sink). If the axions were to equilibrate fully, they would all move very close to the equatorial plane. Because their thermalization rate is not much larger than the Hubble rate, we expect that the axions start to move towards the equator but there is not enough time for the axions to get all localized there. The motion of the axions toward the equatorial plane is another way to see why the big vortex forms.

There is a large and growing literature exploring the hypothesis that the dark matter is a Bose-Einstein condensate of spin zero particles with mass of order $10^{-21} \mathrm{eV}$, or so [15, 16]. When the mass is that small, the de Broglie wavelength of the BEC in galactic halos is large enough (of order kpc) that the wave nature of the BEC has observable effects. In contrast, our proposal is that the dark matter is composed of ordinary QCD axions, or of axion-like particles with properties similar to QCD axions. That QCD axions form a BEC is not an assumption on our part but a consequence of their standard properties. The axion BEC forms because the axions thermalize as a result of their gravitational interactions [3, 5]. The axion BEC behaves differently from WIMPs because it rethermalizes on time scales less than the age of the universe. The process of thermalization is key to understanding the properties of any BEC. It is only by thermalizing that a macroscopically large fraction of degenerate identical bosons go to their lowest energy available state. The process of thermalization is not described by the Gross-Pitaevskii equation. That equation describes the properties of the state that the particles condense into, but not the process by which the particles condense into that state. We emphasize in Section II that vortices appear in a BEC only as part of the process of rethermalization. The Gross-Pitaevskii equation describes the properties of vortices and their motions, but not their appearance.

The outline of this paper is as follows. In Section II, we generalize the rules of statistical mechanics to the case where the many body system conserves angular momentum. We derive the rule that determines the state that a rotating $\mathrm{BEC}$ condenses into. We verify that the rule is consistent with the known behaviour of superfluid ${ }^{4} \mathrm{He}$ and derive the expected behavior of a rotating BEC of quasi-collisionless particles. In Section III, we obtain the expected behavior of an axion BEC about to fall into a galactic gravitational potential well, and the response of baryons and WIMPS to the presence of the axion BEC. In Section IV, we show that the axion BEC provides a solution to the galactic angular problem and derive a minimum fraction of dark matter in axions (37\%) from the typical size of rises in the Milky Way rotation curve attributed to caustic rings. Section V provides a summary.

\section{STATISTICAL MECHANICS OF ROTATING SYSTEMS}

In this section we discuss theoretical issues related to the statistical mechanics of rotating many-body systems. First we generalize the well-known equilibrium Bose-Einstein, Fermi-Dirac and Maxwell-Boltzmann distributions to the case where angular momentum is conserved. Systems which conserve angular momentum are characterized by an angular velocity, in addition to their temperature and chemical potential. Next we discuss the selfgravitating isothermal sphere [17] as a model for galactic halos. We show that the model is a reasonably good description only when the angular momentum is zero. Next we discuss rotating Bose-Einstein condensates (BEC) and obtain the rule that determines the state which 
particles condense into. We analyze the properties of the vortices that must be present in any rotating Bose-Einstein condensate, and discuss the contrasting behaviours of vortices in superfluid ${ }^{4} \mathrm{He}$ and in a fluid of quasi-collisionless particles. Finally, we identify rethermalisation as the mechanism by which vortices appear in a BEC after it has been given angular momentum.

\section{A. Temperature, chemical potential and angular velocity}

A standard textbook result gives the average occupation number $\mathcal{N}_{i}$ of particle state $i$ in a system composed of a huge number of identical particles at temperature $T$ and chemical potential $\mu$ :

$$
\mathcal{N}_{i}=\frac{1}{e^{\frac{1}{T}\left(\epsilon_{i}-\mu\right)}-\sigma}
$$

where $\epsilon_{i}$ is the energy of particle state $i$, and $\sigma=0,+1$ or -1 . If the particles are distinguishable, one must take $\sigma=0$ and the distribution is called Maxwell-Boltzmann. If the particles are bosons, $\sigma=+1$ and the distribution is called Bose-Einstein. If the particles are fermions, $\sigma=-1$ and the distribution is called Fermi-Dirac.

To obtain Eq. (2.1), one considers a system with given total energy $E=\sum_{i} n_{i} \epsilon_{i}$ and given total number of particles $N=\sum_{i} n_{i}$. The $\mathcal{N}_{i}$ are the values of the $n_{i}$ which maximize the entropy [18, 19]. One may repeat this exercise in the case of a system that conserves total angular momentum $L=\sum_{i} n_{i} l_{i}$ about some axis, say $\hat{z}$. Maximizing the entropy for given total energy $E$, total number of particles $N$ and total angular momentum $L$, one finds:

$$
\mathcal{N}_{i}=\frac{1}{e^{\frac{1}{T}\left(\epsilon_{i}-\mu-\omega l_{i}\right)}-\sigma}
$$

where $\omega$ is an angular velocity. The system at equilibrium is characterized by $T, \mu$ and $\omega$. If the total number of particles is not conserved, one must set $\mu=0$. Likewise if total angular momentum is not conserved one must set $\omega=0$.

\section{B. The self-gravitating isothermal sphere revisited}

Consider a huge number of self-gravitating identical classical particles. (Although identical, they are disinguishable by arbitrarily unobtrusive labels.) A particle state is given by its location $(\vec{r}, \vec{v})$ in phase-space. According to Eq. (2.2), the particle density in phase-space is given at thermal equilibrium by

$$
\mathcal{N}(\vec{r}, \vec{v})=\mathcal{N}_{0} e^{-\frac{m}{T}\left[\frac{1}{2} \vec{v} \cdot \vec{v}+\Phi(\vec{r})-\omega \hat{z} \cdot(\vec{r} \times \vec{v})\right]}
$$

where $m$ is the particle mass, $\mathcal{N}_{0} \equiv e^{\frac{\mu}{T}}$, and $\Phi(\vec{r})$ is the gravitational potential. Newtonian gravity is assumed. The gravitational potential satisfies the Poisson equation:

$$
\nabla^{2} \Phi(\vec{r})=4 \pi G m n(\vec{r})
$$

where

$$
n(\vec{r})=\int d^{3} v \mathcal{N}(\vec{r}, \vec{v})
$$


is the physical space density.

When $\omega=0$, Eqs. (2.3) and (2.5) imply

$$
n(\vec{r})=n_{0} e^{-\frac{3}{<v^{2}>} \Phi(\vec{r})}
$$

where $\left\langle v^{2}\right\rangle=\frac{3 T}{m}$ is the velocity dispersion of the particles and $n_{0}=\mathcal{N}_{0}\left(\frac{\left.2 \pi<v^{2}\right\rangle}{3}\right)^{\frac{3}{2}}$. Combining Eqs. (2.4) and (2.6) one obtains

$$
\nabla^{2} \Phi(\vec{r})=4 \pi G m n_{0} e^{-\frac{3}{<v^{2}>} \Phi(\vec{r})}
$$

This equation permits a spherically symmetric ansatz, $\Phi(\vec{r})=\Phi(r)$. It can then be readily solved by numerical integration. The solutions have the form

$$
n(r)=n_{0} d(r / s)
$$

where

$$
s=\left(\frac{<v^{2}>}{12 \pi G m n_{0}}\right)^{\frac{1}{2}}
$$

and $d(x)$ is a unique function with the limiting behaviours: $d(x) \rightarrow 1$ as $x \rightarrow 0$ and $d(x) \rightarrow 2 / x^{2}$ as $x \rightarrow \infty$. A plot of the function $d(x)$ is shown, for example, in Fig. 1 of ref. [20] or Fig. 4.7 of ref. [21]. The function $d(x)$ is often approximated by $\frac{2}{2+x^{2}}$ for convenience. The phase-space distribution

$$
\mathcal{N}(\vec{r}, \vec{v})=\mathcal{N}_{0} e^{-\frac{3}{<v^{2}>} \vec{v} \cdot \vec{v}} d(r / s)
$$

is called an 'isothermal sphere' [17].

The isothermal sphere is often used as a model for galactic halos [22, 23]. As such it has many attractive properties. First, the isothermal sphere model is very predictive since it gives the full phase-space distribution in terms of just two parameters, $\left\langle v^{2}\right\rangle$ and $s$. Second, these two parameters are directly related to observable properties of a galaxy: $\left\langle v^{2}\right\rangle$ is related to the galactic rotation velocity at large radii $v_{\text {rot }}$ by $v_{\text {rot }}=\sqrt{\frac{2}{3}\left\langle v^{2}\right\rangle}$ and $s$ is related to the galactic halo core radius $a$ by $a=\sqrt{2} s$. Third, since $n(r) \propto 1 / r^{2}$ for large $r$, the isothermal model predicts galactic rotation curves to be flat at large $r$. This is consistent with observation. Fourth, since $n(r) \simeq n_{0}$ for small $r$, galactic halos have inner cores where the density is constant. This is also consistent with observation. Fifth, the model is based on a simple physical principle, namely thermalization.

For all its virtues, we do not believe the isothermal model to be a good description of galactic halos. The reason is that present day galactic halos, such as that of the Milky Way, are unlikely to be in thermal equilibrium. If for some unexplained reason the Milky Way halo were in thermal equilibrium today, it would soon leave thermal equilibrium because it accretes surrounding dark matter. The infalling dark matter particles only thermalize on time scales that are much longer than the age of the universe [24, 25]. The flows of infalling dark matter produce peaks in the velocity distribution. A large fraction of the halo, over $90 \%$ in the model of ref. [9], is in cold flows. This disagrees with the smooth Maxwell-Boltzmann distribution, Eq. (2.10), of the isothermal model. The presence of infall flows, with high density contrast in phase-space, has been confirmed by cosmological N-body simulations [26]. 
Here we point to another flaw of the isothermal sphere as a model of galactic halos. Galactic halos acquire angular momentum from tidal torquing. If they are in thermal equilibrium, as the isothermal model supposes, the phase-space distribution must be given by Eq. (2.3) with $\omega \neq 0$. However, Eq. (2.3) is an unacceptably poor description of galactic halos as soon as $\omega \neq 0$. Indeed, Eq. (2.3) may be rewritten

$$
\mathcal{N}(\vec{r}, \vec{v})=\mathcal{N}_{0} e^{-\frac{m}{T}\left[\frac{1}{2}(\vec{v}-\vec{\omega} \times \vec{r})^{2}+\Phi(\vec{r})-\frac{1}{2}(\vec{\omega} \times \vec{r})^{2}\right]}
$$

where $\vec{\omega}=\omega \hat{z}$. Compared to the $\omega=0$ case, the velocity distribution is locally boosted by the rigid rotation velocity $\vec{\omega} \times \vec{r}$. The physical space density is

$$
n(\vec{r})=n_{0} e^{-\frac{m}{T}\left[\Phi(\vec{r})-\frac{1}{2}(\vec{\omega} \times \vec{r})^{2}\right]} \quad .
$$

Substituting this into Eq. (2.4), one obtains

$$
\nabla^{2} \Phi(\vec{r})=4 \pi G m n_{0} e^{\frac{m}{T}\left[-\Phi(\vec{r})+\frac{1}{2} \omega^{2} \rho^{2}\right]} \quad .
$$

where $(\rho, z, \phi)$ are cylindrical coordinates. Eq. (2.13) does not have any solutions for which the density $n(\vec{r})$ goes to zero for large $\rho$. Indeed $d(\vec{r}) \propto e^{\frac{m}{2 T} \omega^{2} \rho^{2}}$ at large $\rho$ unless $\Phi \rightarrow \frac{1}{2} \omega^{2} \rho^{2}$ there. But this implies, through Eq. (2.4), that the density goes to the constant value $\frac{\omega^{2}}{2 \pi G m}$ at large $\rho$. The particles at large $\rho$ have huge bulk motion with average velocity $\vec{\omega} \times \vec{r}$. Thus the rotating isothermal sphere is an object of infinite extent in a state of rigid rotation. This is certainly inconsistent with the properties of galactic halos.

As was discussed by Lynden-Bell and Wood [27], isothermal spheres and self-gravitating systems in general are unstable because their specific heat is negative, i.e. they get hotter when energy is extracted from them. The instability implies a gravo-thermal catastrophe on a time scale which may be inconsistent with the age of galactic halos and thus cause further difficulties in using isothermal spheres as models for galactic halos. The instability of rotating isothermal spheres is discussed in ref. [28].

\section{The rotating Bose-Einstein condensate}

\section{Bose-Einstein condensation}

Bose-Einstein condensation occurs when the following four conditions are satisfied: 1) the system is composed of a huge number of identical bosons, 2) the bosons are highly degenerate, i.e. their average quantum state occupation number is larger than some critical value of order one, 3) the number of bosons is conserved, and 4) the system is in thermal equilibrium. When the four conditions are satisfied a fraction of order one of all the bosons are in the same state.

Let us recall a simple argument [29] why Bose-Einstein condensation occurs. We first set $\omega=0$. For identical bosons, Eq. (2.2) states

$$
\mathcal{N}_{i}=\frac{1}{e^{\frac{1}{T}\left(\epsilon_{i}-\mu\right)}-1}
$$

Let $i=0$ be the ground state, i.e. the particle state with lowest energy. It is necessary that the chemical potential remain smaller than the ground state energy $\epsilon_{0}$ at all times since 
Eq. (2.14) does not make sense for $\epsilon_{i}<\mu$. The total number of particles $N(T, \mu)=\sum_{i} \mathcal{N}_{i}$ is an increasing function of $\mu$ for fixed $T$ since each $\mathcal{N}_{i}$ has that property. Let us imagine that the total number $N$ of particles is increased while $T$ is held fixed. The chemical potential increases till it reaches $\epsilon_{0}$. At that point the total number of particles in excited $(i>0)$ states has its maximum value

$$
N_{\mathrm{ex}}\left(T, \mu=\epsilon_{0}\right)=\sum_{i>0} \frac{1}{e^{\frac{1}{T}\left(\epsilon_{i}-\epsilon_{0}\right)}-1} .
$$

In three spatial dimensions, $N_{\mathrm{ex}}\left(T, \mu=\epsilon_{0}\right)$ is finite [29]. Consider what happens when, at a fixed temperature $T, N$ is made larger than $N_{\text {ex }}\left(T, \mu=\epsilon_{0}\right)$. The only possible system response is for the extra $N-N_{\text {ex }}\left(T, \mu=\epsilon_{0}\right)$ particles to go to the ground state. Indeed the occupation number $\mathcal{N}_{0}$ of that state becomes arbitrarily large as $\mu$ approaches $\epsilon_{0}$ from below. This is the phenomenon of Bose-Einstein condensation.

We may repeat the above argument for $\omega \neq 0$. In this case the chemical potential $\mu$ must remain smaller than the smallest $\eta_{i} \equiv \epsilon_{i}-\omega l_{i}$. The existence of a minimum $\eta_{i}$ is guaranteed because the particle energy $\epsilon_{i}$ always contains a piece that is quadratic in $l_{i}$, namely the kinetic energy associated with motion in the $\hat{\phi}$ direction. Let

$$
\eta_{0} \equiv \min _{i}\left[\epsilon_{i}-\omega l_{i}\right]
$$

and $i=0$ the particle state that minimizes $\eta$. (We are relabeling the states compared to the $\omega=0$ case.) The largest possible number of particles in states $i \neq 0$ at a given temperature $T$ is

$$
N_{\mathrm{ex}}\left(T, \mu=\eta_{0}\right)=\sum_{i \neq 0} \frac{1}{e^{\frac{1}{T}\left(\eta_{i}-\eta_{0}\right)}-1} .
$$

If the total number $N$ of particles is larger than $N_{\text {ex }}\left(T, \mu=\eta_{0}\right)$, the extra $N-N_{\text {ex }}\left(T, \mu=\eta_{0}\right)$ particles go to the $i=0$ state.

\section{Fluid description of the condensed state}

Each particle state is described by a wavefunction $\Psi(\vec{r}, t)$ which satisfies the Schrödinger equation (we suppress for the time being the index $i$ that labels particle states)

$$
i \partial_{t} \Psi=\left[-\frac{1}{2 m} \nabla^{2}+m \Phi(\vec{r}, t)+V(\vec{r}, t)\right] \Psi
$$

where $\Phi(\vec{r}, t)$ is the gravitational potential and $V(\vec{r}, t)$ is the non-gravitational potential energy of the particle in its background. We assume throughout that the particles are nonrelativistic. As is well-known, the probability density $\Psi^{*} \Psi$ and the probability flux density $\frac{1}{m} \operatorname{Im}\left(\Psi^{*} \vec{\nabla} \Psi\right)$ satisfy the continuity equation because the total probability to find the particle someplace is conserved.

When a state is occupied by a huge number $N$ of particles, that state's wavefunction $\Psi(\vec{r}, t)$ describes the properties of a macroscopic fluid. The fluid density is

$$
n(\vec{r}, t)=N \Psi^{*} \Psi
$$


and the fluid flux density is

$$
\vec{j}(\vec{r}, t)=N \frac{1}{m} \operatorname{Im}\left(\Psi^{*} \vec{\nabla} \Psi\right)
$$

They satisfy the continuity equation

$$
\partial_{t} n+\vec{\nabla} \cdot \vec{j}=0
$$

for the reason mentioned at the end of the previous paragraph. The fluid velocity $\vec{v}(\vec{r}, t)$ is defined by $\vec{v} \equiv \frac{1}{n} \vec{j}$. If we write the wavefunction as

$$
\Psi(\vec{r}, t) \equiv B(\vec{r}, t) e^{i \beta(\vec{r}, t)}
$$

then $n=N B^{2}, \vec{j}=N B^{2} \frac{1}{m} \vec{\nabla} \beta$ and hence [29]

$$
\vec{v}(\vec{r}, t)=\frac{1}{m} \vec{\nabla} \beta
$$

When a state is occupied by a huge number $N$ of particles, its wavefunction $\Psi(\vec{r}, t)$ satisfies Eq. (2.18) as does the wavefunction of any other state. However, the gravitational potential $\Phi$ and the potential energy $V$ may have important contributions from the $N$ particles themselves, i.e.

$$
\Phi(\vec{r}, t)=-G m \int d^{3} r^{\prime} \frac{n\left(\vec{r}^{\prime}, t\right)}{\left|\vec{r}-\vec{r}^{\prime}\right|}+\ldots
$$

where the dots indicate other contributions to the gravitational potential, and likewise

$$
V(\vec{r}, t)=\int d^{3} r^{\prime} V_{p}\left(\vec{r}-\vec{r}^{\prime}\right) n\left(\vec{r}^{\prime}, t\right)+\ldots
$$

if the $N$ particles interact pairwise with forces derived from a potential $V_{p}$. Upon substituting Eqs. (2.24) and/or (2.25), Eq. (2.18) takes on a non-linear form. This non-linear form of the Schrödinger equation is called the Gross-Pitaevskii equation.

Eq. (2.18) implies an Euler-type equation for the fluid velocity [29]

$$
\partial_{t} \vec{v}+(\vec{v} \cdot \vec{\nabla}) \vec{v}=-\vec{\nabla} q-\vec{\nabla} \Phi-\frac{1}{m} \vec{\nabla} V
$$

where

$$
q(\vec{r}, t)=-\frac{1}{2 m^{2}} \frac{\nabla^{2} \sqrt{n}}{\sqrt{n}}
$$

Except for the $-\vec{\nabla} q$ term, Eq. (2.26) is the Euler equation for a fluid of classical particles moving in the potentials $\Phi$ and $V$. The $-\vec{\nabla} q$ term is a consequence of the Heisenberg uncertainty principle and accounts, for example, for the intrinsic tendency of a wavepacket to spread. However, the $-\vec{\nabla} q$ term is irrelevant on scales large compared to the de Broglie wavelength. On such large length scales the fluid described by the Schrödinger equation is indistinguishable from a fluid of classical particles moving in the potentials $\Phi$ and $V$. 


\section{Vortices [30, 31]}

It may appear at first that the requirement $\vec{\nabla} \times \vec{v}=0$, implied by Eq. (2.23), disagrees with the principle just stated, since a fluid of particles may have a rotational velocity field whereas the wave description allows apparently only irrotational flow. However, that appearance is deceiving because Eq. (2.23) is valid only where $\Psi \neq 0$. Indeed $\beta$ is not well defined where $\Psi=0$. The following example is instructive

$$
\Psi(\rho, \phi, t)=A J_{l}(k \rho) e^{i l \phi} e^{-i \frac{k^{2}}{2 m} t}
$$

where $J_{l}$ is the Bessel function of index $l$. This wavefunction solves the Schrödinger equation with $\Phi=V=0$. It describes an axially symmetric flow with energy $\frac{k^{2}}{2 m}$ and $z$-component of angular momentum $l$ per particle. The fluid is clearly rotating since Eq.(2.23) implies the velocity field

$$
\vec{v}=\frac{l}{m \rho} \hat{\phi}
$$

The curl of that velocity field vanishes everywhere except on the $z$-axis: $\vec{\nabla} \times \vec{v}=\frac{2 \pi l}{m} \hat{z} \delta^{2}(x, y)$ where $x$ and $y$ are Cartesian coordinates in the plane perpendicular to $\hat{z}$. $\Psi$ vanishes on the $z$-axis if $l \neq 0$. Furthermore, since the Bessel function $J_{l}(s) \simeq 0$ for $s<<l$ [32], the density implied by Eq. (2.28) is tiny for $\rho$ much less than the classical turnaround radius $\rho_{c}=l / k$. Thus, on length scales large compared to $k^{-1}$, the fluid motion described by the wavefunction (2.28) is the same as the motion of a fluid composed of classical particles, in agreement with the principle stated at the end of the previous paragraph.

That the motion of a fluid of cold dark matter particles can be described by a wavefunction was emphasised by Widrow and Kaiser some twenty years ago [33]. Ordinary (non BEC) cold dark matter particles have irrotational flow because their rotational modes have been suppressed by the expansion of the universe and, when density perturbations start to grow, Eq.(2.26) implies $\vec{\nabla} \times \vec{v}=0$ at all times if $\vec{\nabla} \times \vec{v}=0$ initially [6]. The previous paragraph emphasizes that a fluid of cold dark matter particles can be described by a wavefunction whether or not the velocity field is irrotational. The only requirement is that the wavefunction vanish on a set of lines, called vortices, if the velocity field is rotational.

Each vortex carries an integer number of units of angular momentum, $l$ units in the example of Eq. (2.28). The following rule applies. Let $\mathcal{C}[\Gamma]$ be the circulation of the velocity field along a closed path $\Gamma$

$$
\mathcal{C}[\Gamma] \equiv \oint_{\Gamma} d \vec{r} \cdot \vec{v}(\vec{r}, t)
$$

If the fluid is represented by a wave, Eq. (2.23) implies

$$
\mathcal{C}[\Gamma]=\frac{\Delta \beta}{m} \equiv \frac{2 \pi}{m} l[\Gamma]
$$

where $\Delta \beta$ is the change of the phase $\beta$ when going around $\Gamma$. Since the wavefunction is single valued, $l[\Gamma] \equiv \frac{1}{2 \pi} \Delta \beta$ is an integer. The surface enclosed by a closed path with nonzero circulation must be traversed by vortices whose units of angular momentum add up to $l[\Gamma]$. Another rule is that as long as the fluid is described by a wavefunction $\Psi(\vec{r}, t)$, vortices cannot appear spontaneously in the fluid. They can only move about. Indeed, consider an arbitrary closed path $\Gamma$. The total number of vortices encircled by $\Gamma$, counting a vortex with angular momentum $l$ as $l$ vortices, is determined by the constraint of Eq. (2.31). The only 
way the RHS of that equation can change is by having the wavefunction $\Psi$ vanish somewhere on $\Gamma$ and letting a vortex cross that curve. Finally, a third rule: vortices must follow the motion of the fluid. This is a corollary of Kelvin's theorem which states that, in gradient flow - i.e. if the RHS of Euler's equation is a gradient, as is the case for Eq. (2.26) - the circulation of the velocity field along a closed path that moves with the fluid is constant in time.

\section{Superfluid ${ }^{4} \mathrm{He}$}

Let us see how the above considerations apply first to the case of superfluid ${ }^{4} \mathrm{He}$, and next to the case of a collisionless fluid. Of course, we have nothing new to say about superfluid ${ }^{4} \mathrm{He}$, which we discuss merely to build confidence in the general approach described so far. We found that, when Bose-Einstein condensation occurs, a macroscopically large number of particles, say $N$, condense into the state with lowest $\eta=\epsilon-\omega l$. We have, in terms of the quantities defined earlier,

$$
\begin{aligned}
\epsilon & =\int d^{3} r \Psi^{*}\left(-\frac{1}{2 m} \nabla^{2}+m \Phi+V\right) \Psi \\
& =\frac{1}{N} \int d^{3} r n\left[\frac{m}{2} \vec{v} \cdot \vec{v}+m(q+\Phi)+V\right]
\end{aligned}
$$

and

$$
\begin{aligned}
l & =\int d^{3} r \Psi^{*} \hat{z} \cdot\left(\vec{r} \times \frac{1}{i} \vec{\nabla}\right) \Psi=\int d^{3} r \Psi^{*} \frac{1}{i} \frac{\partial}{\partial \phi} \Psi \\
& =\frac{1}{N} \int d^{3} r n m \hat{z} \cdot(\vec{r} \times \vec{v}) .
\end{aligned}
$$

He atoms have an interparticle potential $V_{p}$ that describes forces that are strongly repulsive at short range and weakly attractive at long range. In the liquid state, the average interatomic distance is of order the atom size. Thus the density of superfluid ${ }^{4}$ He has an approximately constant value $n_{0}$. In that case

$$
\epsilon \simeq \frac{n_{0}}{N} \int d^{3} r \frac{m}{2} \vec{v} \cdot \vec{v} \quad, \quad l \simeq \frac{n_{0}}{N} \int d^{3} r m \hat{z} \cdot(\vec{r} \times \vec{v})
$$

and therefore

$$
\eta \simeq \frac{n_{0} m}{2 N} \int d^{3} r\left[\left(v_{z}\right)^{2}+\left(v_{\rho}\right)^{2}+\left(v_{\phi}-\omega \rho\right)^{2}-\omega^{2} \rho^{2}\right]
$$

$\eta$ is minimized when $\vec{v}=\omega \hat{z} \times \vec{r}$. So, when superfluid ${ }^{4}$ He carries angular momentum, it is in a state of rigid rotation when viewed on length scales large compared to the de Broglie wavelength. Because rigid rotation implies that the velocity field has non-zero curl, vortices are present. The vortices are parallel to the $z$-axis and their number density per unit area is

$$
\frac{m}{2 \pi} \hat{z} \cdot(\vec{\nabla} \times \vec{v})=\frac{m \omega}{\pi}
$$

according to Eqs. (2.30) and (2.31). The transverse size of a vortex is determined by balancing the competing effects of $-\vec{\nabla} q$ and $-\frac{1}{m} \vec{\nabla} V$ in Eq. (2.26). $-\vec{\nabla} q$ tends to increase the 
transverse size of the vortex whereas $-\frac{1}{m} \vec{\nabla} V$ tends to decrease it assuming, as is the case in superfluid ${ }^{4} \mathrm{He}$, that the interparticle interactions are repulsive at short distances. The outcome determines the transverse size of a vortex to be a characteristic length $\xi$, called the 'healing length' [29]. For the interparticle potential

$$
V_{p}\left(\vec{x}-\vec{x}^{\prime}\right)=U_{0} \delta^{3}\left(\vec{x}-\vec{x}^{\prime}\right)
$$

the healing length is

$$
\xi=\frac{1}{\sqrt{2 m n_{0} U_{0}}} .
$$

The transverse size of a $l$-vortex, i.e. a vortex that carries $l$ units of angular momentum, is $l \xi$. Indeed the behaviour at short distances to the vortex center is the same as in Eq. (2.28) with $k$ replaced by $\xi^{-1}$. The cross-sectional area of a $l$-vortex is therefore of order $\pi \xi^{2} l^{2}$. Also its energy per unit length [29] is proportional to $l^{2}$. The vortices repel each other because a $l$-vortex has more energy per unit length than $l 1$-vortices. The lowest energy configuration for given angular momentum per unit area is a triangular lattice of parallel 1-vortices [34]. Such triangular vortex arrays were observed in superfluid ${ }^{4} \mathrm{He}$ [35] and in Bose-Einstein condensed gases [36].

\section{Quasi-collisionless particles}

We finally arrive at the object of our interest, a Bose-Einstein condensate of quasicollisionless particles. The particles cannot be exacly collisionless since they must thermalize to form a BEC and they can only thermalize if they interact. However, the interaction by which the particles thermalize can be arbitrarily weak since the thermalization may, in principle, occur on an arbitrarily long time scale. In that limit we may set $V=0$ in Eq. (2.18). For the sake of definiteness we set the gravitational field $\Phi=0$ as well. We have then

$$
\begin{aligned}
\eta & =\int d^{3} r \Psi^{*}\left(-\frac{1}{2 m} \nabla^{2}-\frac{\omega}{i} \frac{\partial}{\partial \phi}\right) \Psi \\
& =\frac{1}{N} \int d^{3} r n\left[\frac{m}{2} \vec{v} \cdot \vec{v}+m q-m \omega \hat{z} \cdot(\vec{r} \times \vec{v})\right] .
\end{aligned}
$$

If one approximates the Bose-Einstein condensate as a fluid of classical particles (setting $q=0$ and taking $n(\vec{r})$ and $\vec{v}(\vec{r})$ to be independent variables), the state of lowest $\eta$ is one of rigid rotation with angular velocity $\omega \hat{z}$ and all particles placed as far from the $z$-axis as possible. For the reasons stated earlier, this is a good approximation only on length scales large compared to the BEC de Broglie wavelength. To obtain the exact BEC state, one must solve the eigenvalue problem

$$
\left(-\frac{1}{2 m} \nabla^{2}-\omega \frac{1}{i} \frac{\partial}{\partial \phi}\right) \Psi_{i}=\eta_{i} \Psi_{i}
$$

The BEC state is then $\Psi_{0}$ such that $\eta_{0}=\min _{i} \eta_{i}$. Since by assumption the system conserves angular momentum, the operators $-\frac{1}{2 m} \nabla^{2}$ and $\frac{1}{i} \frac{\partial}{\partial \phi}$ are simultaneously diagonalizable. Thus $(i=k l)$

$$
\Psi_{k l}(z, \rho, \phi)=A_{k l}(z, \rho) e^{i l \phi}, \quad \eta_{k l}=\epsilon_{k l}-\omega l
$$


and

$$
-\frac{1}{2 m}\left(\frac{\partial^{2}}{\partial z^{2}}+\frac{1}{\rho} \frac{\partial}{\partial \rho} \rho \frac{\partial}{\partial \rho}-\frac{l^{2}}{\rho^{2}}\right) A_{k l}=\epsilon_{k l} A_{k l} \quad .
$$

Let us consider the particular example of a BEC contained in a cylinder of radius $R$ and height $h$. In this case, the operators $-\frac{1}{2 m} \nabla^{2}, \frac{1}{i} \frac{\partial}{\partial \phi}$ and $\left(\frac{1}{i} \frac{\partial}{\partial z}\right)^{2}$ are simultaneously diagonalized by

$$
\Psi_{l p n}=e^{i l \phi} \sin \left(\frac{\pi p}{h} z\right) J_{l}\left(x_{l n} \frac{\rho}{R}\right)
$$

where $l=0, \pm 1, \pm 2, \ldots, p=1,2,3, \ldots, n=1,2,3, \ldots$, and $x_{l n}$ is the $n^{\text {th }}$ root of $J_{l}(x)$ with $x_{l 1}<x_{l 2}<x_{l 3}<\ldots$. Since

$$
\epsilon_{l p n}=\frac{1}{2 m}\left[\left(\frac{\pi p}{h}\right)^{2}+\left(\frac{x_{l n}}{R}\right)^{2}\right]
$$

$\eta_{l p n}$ is minimized by setting $p=n=1$ and $l=l_{0}$ where $l_{0}$ minimizes $\frac{1}{2 m}\left(\frac{x_{l 1}}{R}\right)^{2}-\omega l$. For large $l$, the first zero of $J_{l}[32]$

$$
x_{l 1} \simeq l+1.85575 l^{\frac{1}{3}}+\mathcal{O}\left(l^{-\frac{1}{3}}\right) .
$$

Hence

$$
l_{0}=m R^{2} \omega\left[1-2.47433\left(m R^{2} \omega\right)^{-\frac{2}{3}}+\mathcal{O}\left(m R^{2} \omega\right)^{-\frac{4}{3}}\right] \quad .
$$

When the BEC is approximated as a fluid of classical particles, the BEC state is rigid rotation with all the particles located at $\rho=R$, not necessarily in a uniform way. In the actual BEC state the particles are, for large $l$, uniformly located just inside the $\rho=R$ surface, in a film of thickness $\delta \rho \sim R l^{-\frac{2}{3}}$.

Unlike the case of superfluid ${ }^{4} \mathrm{He}$, vortices in a collisionless BEC attract each other. Indeed the lowest energy state for given total angular momentum $l$ is a single $l$-vortex with transverse size as large as possible. We may imagine turning off the interparticle repulsion in superfluid ${ }^{4}$ He placed in a cylindrical container. Starting with a triangular array of $l$ parallel 1 -vortices but progressively decreasing $U_{0}$, the vortices grow in transverse size till they join into a single $l$-vortex and all matter is uniformly concentrated near the $\rho=R$ surface.

\section{Thermalization and vortex formation}

We emphasized that vortices cannot appear spontaneously in a fluid that is described by a (single) wavefunction $\Psi$. The Gross-Pitaevskii equation can only describe the motion of vortices, not their appearance. How then do the vortices appear? The vortices appear when the bosons move between different particle states, some of which have vortices and some of which don't. When angular momentum is given to a BEC that is free of vortices, it will at first remain free of vortices even though it carries angular momentum. The vortices only appear when the BEC rethermalizes and the particles go to the new lowest energy state consistent with the angular momentum the BEC received.

Consider, for example, a BEC of spin zero particles in a cylindrical volume. The wavefunctions of the particle states are given by Eq. (2.43). The Hamiltonian is the sum of free and interacting parts: $H=H_{0}+H_{1}$. The free Hamiltonian is:

$$
H_{0}=\sum_{l p n} \epsilon_{l p n} a_{l p n}^{\dagger} a_{l p n}
$$


where $a_{l p n}$ and $a_{l p n}^{\dagger}$ are annihilation and creation operators satisfying canonical commutation relations and generating a Fock space in the usual fashion. We assume that the interaction has the general form

$$
H_{1}=\sum_{i, i^{\prime}, i^{\prime \prime}, i^{\prime \prime \prime}} \frac{1}{4} \Lambda_{i^{\prime \prime} i^{\prime \prime \prime}}^{i i^{\prime}} a_{i^{\prime \prime \prime}}^{\dagger} a_{i^{\prime \prime}}^{\dagger} a_{i^{\prime}} a_{i}
$$

where $i \equiv l p n, i^{\prime} \equiv l^{\prime} p^{\prime} n^{\prime}$ and so forth, so that the total number of particles

$$
N=\sum_{l p n} a_{l p n}^{\dagger} a_{l p n}
$$

is conserved. In addition we require that

$$
\Lambda_{i^{\prime \prime} i^{\prime \prime \prime}}^{i i^{\prime}}=0 \quad \text { unless } \quad l+l^{\prime}=l^{\prime \prime}+l^{\prime \prime \prime}
$$

so that the total angular momentum

$$
L=\sum_{l p n} l a_{l p n}^{\dagger} a_{l p n}
$$

is conserved as well. The interaction $H_{1}$ causes the system to thermalize on some time scale $\tau=\frac{1}{\Gamma}$. Ref. [5] estimates the thermalization rate $\Gamma$ of cold dark matter axions through their $\lambda \phi^{4}$ and gravitational self-interactions. The relevant thing for our discussion here is only that there is a finite time scale $\tau=\frac{1}{\Gamma}$ over which the system thermalizes.

Let us suppose that $N$ particles are in thermal equilibrium in the cylinder with $\omega=0$ and temperature $T$ well below the critical temperature for Bose-Einstein condensation. A macroscopically large number $N_{0}$ of particles are in the ground state $(l, p, n)_{0}=(0,1,1)$, which we label $i=0$ for short. The remaining $N-N_{0}$ particles are in excited $(i \neq 0)$ states. The vorticity of each state equals its $l$ quantum number. The $N_{0}$ particles in the ground state form a fluid with zero vorticity. Many excited states carry vorticity but their occupation numbers are small compared to $N_{0}$. The particles in excited states merely constitute a gas at temperature $T$. Let us suppose that the fluid is then given some angular momentum. This can be done, for example, by having a large mass $M$ which gravitationally attracts the particles in the cylinder go by, producing a time-dependent potential energy $V_{\text {ext }}(\vec{r}, t)$. We assume for the sake of definiteness that the mass $M$ passes by the cylinder on a time scale $\tau_{M}$ which is much shorter than the thermal relaxation time scale $\tau$. While the mass $M$ passes by, each $\phi$ particle stays in whatever state it was in to start with since the interaction $H_{1}$ that allows particles to jump between states is, by assumption, too feeble to have any effect on the $\tau_{M}$ time scale. The wavefunction of each state satisfies the time-dependent Schrödinger equation:

$$
i \partial_{t} \Psi_{i}(\vec{r}, t)=\left[-\frac{1}{2 m} \nabla^{2}+V_{\mathrm{ext}}(\vec{r}, t)\right] \Psi_{i}(\vec{r}, t)
$$

with the initial condition $\Psi_{i}(\vec{r}, t=-\infty)=\Psi_{i}(\vec{r})$. Although each $\Psi_{i}(\vec{r}, t)$ changes in time, for the reasons given earlier, its vorticity does not. Therefore, just after the mass $M$ has passed, the macroscopic fluid described by $\Psi_{0}(\vec{r}, t)$ has no vorticity although it generally has angular momentum. After a time of order $\tau$, the $N$ particles acquire a thermal distribution, Eq. (2.2) with $\sigma=+1$, consistent with the total number of particles $N$, the angular momentum $L$ acquired from the passing mass and total energy $E$ including some energy acquired from 
the passing mass. Assuming the temperature is still below the critical temperature for Bose-Einstein condensation, a macroscopically large number of particles are in the state $(l, p, n)_{0}^{\prime}=\left(l_{0}^{\prime}, 1,1\right)$ with $l_{0}^{\prime}$ given by Eq. (2.46). That state describes a fluid which carries a single vortex with $l_{0}^{\prime}$ units of angular momentum.

\section{AXIONS, BARYONS AND WIMPS}

In this section we apply the considerations of Section II to dark matter axions when they are about to fall into a galactic gravitational potential well. We also discuss the behavior, in the presence of dark matter axions, of baryons and of a possible ordinary cold dark matter component made of weakly interacting massive particles (WIMPs) and/or sterile neutrinos. First we discuss the axions by themselves, ignoring the other particles.

\section{A. Axions}

Axions behave differently from ordinary cold dark matter particles, such as WIMPs or sterile neutrinos, on time scales long compared to their thermalization time scale $\tau \equiv \frac{1}{\Gamma}$ because on time scales long compared to $\tau$ the axions form a BEC and almost all axions go to their lowest energy available state [3, 5]. Ordinary cold dark matter particles do not do this.

It may be useful to clarify the notion of lowest energy available state. Thermalization involves interactions. By lowest energy available state we mean the lowest energy state that can be reached by the thermalizing interactions. In general the system has states of yet lower energy. For example, and at the risk of stating the obvious, when a beaker of superfluid ${ }^{4} \mathrm{He}$ is sitting on a table, the condensed atoms are in their lowest energy available state. This is not their absolute lowest energy state since the energy of the condensed atoms can be lowered by placing the beaker on the floor.

Axions behave in the same way as ordinary cold dark matter on time scales short compared to their thermalization time scale $\tau$ [3]. So, to make a distinction between axions and ordinary cold dark matter it is necessary to observe the dark matter on time scales long compared to $\tau$. The critical question is then: what is the thermalization time scale $\tau$ ?

\section{Axion thermalization}

The relaxation rate of axions through gravitational self-interactions is of order [3, 5, 37]

$$
\Gamma \sim 4 \pi G n m^{2} \ell^{2}
$$

where $n$ and $m$ are their density and mass, and $\ell \equiv \frac{1}{\delta p}$ their correlation length. $\delta p$ is their momentum dispersion. A heuristic derivation of Eq. (3.1) is as follows. If the axions have density $n$ and correlation length $\ell$, they produce gravitational fields of order $g \sim 4 \pi G n m \ell$. Those fields completely change the typical momentum $\delta p$ of axions in a time $\frac{\delta p}{g m}$. $\Gamma$ is the inverse of that time. To estimate the axion relaxation time today, let us substitute $n m \simeq 0.23 \cdot 10^{-29} \mathrm{gr} / \mathrm{cc}$ (the average dark matter density today), $m \simeq 10^{-5} \mathrm{eV}$ (a typical mass for dark matter axions) and $\ell \simeq 2 \cdot 10^{-7} \mathrm{sec} \frac{\mathrm{GeV}}{10^{-4} \mathrm{eV}}=0.6 \cdot 10^{17} \mathrm{~cm}$ (the horizon during the QCD phase transition, stretched by the universe's expansion until today). This yields a 
relaxation time $\tau$ of order $10^{5}$ years, much shorter than the present age of the universe. So dark matter axions formed a BEC a long time ago already. It is found in refs. [3, 5] that the axions first thermalize and form a BEC when the photon temperature is approximately

$500 \mathrm{eV}\left(\frac{f_{a}}{10^{12} \mathrm{GeV}}\right)^{\frac{1}{2}}$ where $f_{a}$ is the axion decay constant. After the axions form a BEC their correlation length $\ell$ increases until it is of order the horizon since the BEC size is limited only by causality.

It may seem surprising that axions thermalize as a result of their gravitational selfinteractions since gravitational interactions among particles are usually negligible. Dark matter axions are an exception because the axions occupy in huge numbers a small number of states (the typical quantum state occupation number is $10^{61}$ ) and those states have enormous correlation lengths, as was just discussed.

It has been claimed [3-5] that the dark matter is axions, at least in part, because axions explain the occurrence of caustic rings of dark matter in galactic halos. For the explanation to succeed it is necessary that the axions that are about to fall onto a galaxy thermalize sufficiently fast that they almost all go to the lowest energy available state consistent with the angular momentum they acquired from neighboring protogalaxies by tidal torquing [38]. Heuristically, the condition is [5]

$$
4 \pi G n m^{2} \ell>\dot{p}=m \dot{v}
$$

where $\dot{v}$ is the acceleration necessary for the axions to remain in the lowest energy state as the tidal torque is applied. Here $\ell$ must be taken to be of order the size of the system, i.e. some fraction of the distance between neighboring protogalaxies. It was found in ref. [5] that the inequality (3.2) is satisfied by a factor of order 30 - i.e. that its LHS is of order 30 times larger than its RHS - independently of the system size.

\section{Caustic rings}

The evidence for caustic rings is summarized in ref. [9]. It is accounted for if the angular momentum distribution of the dark matter particles on the turnaround sphere of a galaxy is given by

$$
\vec{l}(\hat{n}, t)=m j_{\max } \hat{n} \times(\hat{z} \times \hat{n}) \frac{R(t)^{2}}{t}
$$

where $t$ is time since the Big Bang, $R(t)$ is the radius of the turnaround sphere, $\hat{z}$ the galactic rotation axis, $\hat{n}$ the unit vector pointing to an arbitrary point on the turnaround sphere, and $j_{\max }$ a dimensionless parameter that characterizes the amount of angular momentum the particular galaxy has. The turnaround sphere is defined as the locus of particles which have zero radial velocity with respect to the galactic center for the first time, their outward Hubble flow having just been arrested by the gravitational pull of the galaxy. Eq. (3.3) states that the particles on the turnaround sphere rotate rigidly with angular velocity vector $\vec{\omega}=\frac{j_{\max }}{t} \hat{z}$. The time-dependence of the turnaround radius is predicted by the self-similar infall model [39] to be $R(t) \propto t^{\frac{2}{3}+\frac{2}{9 \epsilon}}$. The parameter $\epsilon$ is related to the slope of the evolved power spectrum of density perturbations on galaxy scales [40]. This implies that $\epsilon$ is in the range 0.25 to 0.35 [10]. The evidence for caustic rings is consistent with that particular range of values of $\epsilon$.

Each property of the angular momentum distribution given in Eq. (3.3) maps onto an observable property of the inner caustics of galactic halos: the rigid rotation implied by the 
factor $\hat{n} \times(\hat{z} \times \hat{n})$ causes the inner caustics to be rings of the type described in refs. [6 8 ] the value of $j_{\max }$ determines their overall size, and the $\frac{R(t)^{2}}{t}$ time dependence causes, in the stated $\epsilon$ range, the caustic radii $a_{n}$ to be proportional to $1 / n(n=1,2,3 \ldots)$. The prediction for the caustic radii is

$$
a_{n} \simeq \frac{40 \mathrm{kpc}}{n}\left(\frac{v_{\mathrm{rot}}}{220 \mathrm{~km} / \mathrm{s}}\right)\left(\frac{j_{\max }}{0.18}\right)
$$

where $v_{\text {rot }}$ is the galactic rotation velocity. To account for the evidence for caustic rings, axions must explain Eq. (3.3) in all its aspects. We now show, elaborating the arguments originally given in ref.[4], that axions do in fact account for each factor on the RHS of Eq. (3.3).

$$
\text { 3. } \hat{n} \times(\hat{z} \times \hat{n})
$$

Consider a comoving spherical volume of radius $S(t)$ centered on a protogalaxy. At early times $S(t)=a(t) S$ where $a(t)$ is the cosmological scale factor. At later times $S(t)$ deviates from Hubble flow as a result of the gravitational pull of the protogalactic overdensity. At some point it reaches its maximum value. At that moment it equals the galactic turnaround radius. $S$ is taken to be of order but smaller than the distance to the nearest protogalaxy of comparable size, say one third of that distance. In the absence of angular momentum, the axions have a purely radial motion described by a wavefuntion $\Psi(r, t)=U(r, t)$ where $r$ is the radial coordinate relative to the center of the sphere. When angular momentum is included the radial motion is modified at small radii by the introduction of an angular momentum barrier. This modification of the radial motion is relatively unimportant and we neglect it. The wavefunctions of the states that the axions occupy are thus taken to be

$$
\Psi_{l, p}(r, \theta, \phi, t)=U(r, t) A_{l, p}(\theta, \phi)
$$

where $\theta$ and $\phi$ are the usual spherical angular coordinates $(0 \leq \theta \leq \pi, 0 \leq \phi<2 \pi)$, and $l$ and $p$ are quantum numbers. $l$ is as before the eigenvalue of the $z$-component of angular momentum. The $z$-direction is the direction of the total angular momentum acquired inside the sphere as a result of tidal torquing. We will see below that that direction is time independent. $p$ is an additional quantum number, associated with motion in $\theta$. We normalize $U(r, t)$ and the various $A(\theta, \phi)$ such that

$$
\int_{0}^{S(t)} r^{2} d r|U(r, t)|^{2}=\int_{0}^{\pi} \sin \theta d \theta \int_{0}^{2 \pi} d \phi|A(\theta, \phi)|^{2}=1 .
$$

We suppress the quantum numbers $l$ and $p$ henceforth.

According to Eq. (3.2) the axions thermalize on a time scale $\tau$ that is short compared to the age of the universe. Hence we expect most axions to keep moving to the state of lowest $\eta=\epsilon-\omega(t) l$. The angular frequency $\omega$ is time dependent since the angular momentum is growing by tidal torquing and the moment of inertia is increasing due to the expansion of the volume under consideration. We have

$$
\begin{aligned}
\epsilon & =\int_{r<S(t)} d^{3} x \Psi^{*}\left[-\frac{1}{2 m} \nabla^{2}+m \Phi(r, t)\right] \Psi \\
& =\int_{0}^{S(t)} r^{2} d r U^{*}\left[-\frac{1}{2 m r^{2}} \frac{\partial}{\partial r} r^{2} \frac{\partial}{\partial r}+m \Phi(r, t)\right] U
\end{aligned}
$$




$$
+\frac{1}{2 I(t)} \int d \Omega A^{*}\left[-\frac{1}{\sin \theta} \frac{\partial}{\partial \theta} \sin \theta \frac{\partial}{\partial \theta}-\frac{1}{\sin ^{2} \theta} \frac{\partial^{2}}{\partial \phi^{2}}\right] A
$$

where

$$
\frac{1}{I(t)}=\frac{1}{m} \int_{0}^{S(t)} d r|U(r, t)|^{2} .
$$

$I(t)$ is similar to a moment of inertia but differs from the usual definition because the volume to which it refers is not rotating like a rigid body in three dimensions. Eq. (3.5) implies instead that each spherical shell of that volume rotates with the same angular momentum distribution. The associated angular velocities vary with shell radius $r$ as $r^{-2}$. The inner shells rotate faster than the outer shells because all shells have the same angular momentum distribution.

We take the gravitational potential $\Phi$ to be spherically symmetric. The first term on the RHS of Eq. (3.7) is then independent of the angular variables and irrelevant to what follows. We will ignore it henceforth. Since

$$
l=\int d \Omega A^{*} \frac{1}{i} \frac{\partial}{\partial \phi} A
$$

we have

$$
\begin{aligned}
\eta= & \int d \Omega A^{*}\left[\frac{1}{2 I(t)}\left(-\frac{1}{\sin \theta} \frac{\partial}{\partial \theta} \sin \theta \frac{\partial}{\partial \theta}-\frac{1}{\sin ^{2} \theta} \frac{\partial^{2}}{\partial \phi^{2}}\right)-\omega(t) \frac{1}{i} \frac{\partial}{\partial \phi}\right] A \\
= & \int d \Omega A^{*}\left[\frac{1}{2 I(t)}\left(-\frac{1}{\sin \theta} \frac{\partial}{\partial \theta} \sin \theta \frac{\partial}{\partial \theta}+\frac{1}{\sin ^{2} \theta}\left(\frac{1}{i} \frac{\partial}{\partial \phi}-\omega(t) I(t) \sin ^{2} \theta\right)^{2}\right)\right. \\
& \left.\quad-\frac{1}{2} \omega^{2}(t) I(t) \sin ^{2} \theta\right] A \\
= & \int d \Omega\left[\frac{1}{2 I(t)}\left|\frac{d A}{d \theta}\right|^{2}-\frac{1}{2} \omega^{2}(t) I(t) \sin ^{2} \theta|A|^{2}\right. \\
& \left.\quad+\frac{1}{2 I(t) \sin ^{2} \theta}\left|\left(\frac{1}{i} \frac{\partial}{\partial \phi}-\omega(t) I(t) \sin ^{2} \theta\right) A\right|^{2}\right] .
\end{aligned}
$$

The $\phi$ dependence of $A$ that minimizes $\eta$ is

$$
A(\theta, \phi, t)=\Theta(\theta, t) e^{i \omega(t) I(t) \sin ^{2} \theta \phi} \quad .
$$

However that exact $\phi$ dependence is not allowed because the wavefunction must be singlevalued. Instead we have

$$
A(\theta, \phi, t) \simeq \Theta(\theta, t) e^{i \omega(t) I(t) \sin ^{2} \theta \phi}
$$

by which we mean that $A(\theta, \phi, t)$ is as given in Eq. (3.11) except for the insertion of small defects (vortices) that allow $A$ to be single-valued. The vortices are discussed below.

After Eq. (3.12) is satisfied, we have

$$
\eta \simeq \int d \Omega\left[\frac{1}{2 I(t)}\left|\frac{d \Theta}{d \theta}\right|^{2}-\frac{1}{2} \omega^{2}(t) I(t) \sin ^{2} \theta|\Theta|^{2}\right] .
$$

$\eta$ is further minimized by having $\Theta$ peaked at $\theta=\frac{\pi}{2}$, i.e. at the equator. The width of the peak is of order $\delta \theta \sim \frac{1}{\sqrt{\omega I}}$. Eq. (3.12) shows that $L \equiv \omega I$ is the angular momentum per particle in the galactic plane. A typical value is

$$
L \sim\left(500 \frac{\mathrm{km}}{\mathrm{s}}\right)(10 \mathrm{kpc}) m \simeq 2.6 \cdot 10^{19}\left(\frac{m}{10^{-5} \mathrm{eV}}\right)
$$


Therefore in their state of lowest $\eta$ the axions are almost all within a very small angular distance, of order $10^{-10}$ radians, from the galactic plane.

The state just described is the state most axions would be in after a sufficiently long period of thermalization. Because the thermalization criterion of Eq. (3.2) is only satisfied by a factor of order 30 we expect that, although the axions start to move towards the equator, there is not enough time for all the axions to get localized there. We expect the system to behave as follows. As the axions acquire angular momentum they go to a state, described by Eqs. (3.5) and (3.12), in which each spherical shell rotates rigidly with angular velocity proportional to $r^{-2}$ where $r$ is the shell radius. The axion velocity field is

$$
\vec{v} \simeq v_{r} \hat{r}+v_{\theta} \hat{\theta}+\frac{1}{m r} L(t) \sin \theta \hat{\phi}
$$

The $\simeq$ sign indicates that the LHS and RHS equal each other except for the presence of vortices. The vortices have direction and density per unit surface given by [see Eqs. (2.30) and (2.31) ]

$$
\frac{m}{2 \pi} \vec{\nabla} \times \vec{v} \simeq \vec{\nabla} \times\left(\frac{L(t)}{m r} \sin \theta \hat{\phi}\right)=\frac{L(t)}{\pi r^{2}} \cos \theta \hat{r}
$$

They point in the radial direction, and are more dense near the poles than near the equator. The total vortex number penetrating the northern hemisphere is

$$
\int_{0}^{2 \pi} d \phi \int_{0}^{\frac{\pi}{2}} \sin \theta d \theta r^{2} \frac{m}{2 \pi} \vec{\nabla} \times \vec{v}=L \quad .
$$

As discussed in Section II, axion vortices attract each other. When two vortices combine, their diameters are added. (Two vortices of equal diameter, and hence of equal crosssectional area, combine into a vortex with four times that cross-sectional area). Assuming that a fraction of order one of all the vortices combine with one another, a huge vortex appears along the $\hat{z}$ axis. We will refer to it as the 'big vortex'. The intersection of the big vortex with the galactic plane is a circle whose radius $a^{\prime} \simeq \frac{L^{\prime}}{k}$ where $k$ is the momentum of axions in the equatorial plane at their closest approach to the galactic center and $L^{\prime}$ is the angular momentum carried by the big vortex. The distance of closest approach to the galactic center of axions in the equatorial plane is $a=\frac{L}{k}$. $a$ is also the radius of the caustic ring made by the axions as they fall through the galaxy for the first time. Because of incomplete thermalization, we expect that some fraction of the vortices have not joined the big vortex, implying that $L^{\prime}<L$ and hence $a^{\prime}<a$.

The factor $\hat{n} \times(\hat{z} \times \hat{n})$ in the angular momentum distribution on the turnaround sphere, Eq. (3.3), is thus accounted for by the fact that the axions on the turnaround sphere rotate rigidly. After turnaround there is not enough time for further thermalization and whatever further thermalization may occur would not make an appreciable difference. Thus, after turnaround, the axions fall in and out of the galaxy like ordinary cold collisionless particles but they do so with net overall rotation whereas ordinary cold dark matter falls in with an irrotational velocity field [6].

Why and how the angular momentum distribution of Eq. (3.3) yields caustic rings is explained in refs. [8, 9]. However those papers, written before the discovery of Bose-Einstein condensation of dark matter axions [3], assume that the infall is isotropic, i.e. that the mass falling onto the galaxy per unit time and unit solid angle $\frac{d M}{d \Omega d t}$ does not depend on $\theta$ (nor on $\phi)$. The above discussion suggests that this assumption should be modified since a big 
vortex is now expected along the $\hat{z}$ axis, implying that the infall rate is suppressed near $\theta=0$ and $\pi$. In the self-similar infall model the total mass $M$ of the halo grows as $t \frac{2}{3 \epsilon}$ [39]. Therefore $\frac{d M}{d \Omega d t}=\frac{M}{6 \pi \epsilon t}$ in the isotropic case. We replace this with

$$
\frac{d M}{d \Omega d t}(\theta, t)=N_{v}(\sin \theta)^{v} \frac{M}{6 \pi \epsilon t}
$$

where $v$ (lower case upsilon, not to be confused with $v$ the magnitude of velocity) is a parameter describing the size of the big vortex. The normalization factor

$$
N_{v}=\frac{\Gamma(v+2)}{2^{v}\left(\Gamma\left(\frac{v}{2}+1\right)\right)^{2}}
$$

is such that the total infall rate, integrated over solid angle, remains the same as before. The new model for the infall rate, Eq. (3.18), does not change the prediction that the inner caustics are rings nor the prediction, Eq. (3.4), for the caustic ring radii [41]. It does however imply (for large $v$ ) that the caustic rings are more prominent than in the isotropic infall case since the axions fall in preferentially along the galactic plane. This will be discussed in Section IV.

$$
\text { 4. } \frac{R(t)^{2}}{t}
$$

We now show, repeating the argument of ref. [4], that the $\frac{R(t)^{2}}{t}$ time dependence on the RHS of Eq. (3.3) follows from tidal torque theory in linear order of perturbation theory. Consider again the comoving sphere of radius $S(t)$ introduced above. The total gravitational torque applied to the volume $V(t)$ of the sphere is

$$
\vec{\tau}(t)=\int_{V(t)} d^{3} r \delta \rho(\vec{r}, t) \vec{r} \times(-\vec{\nabla} \phi(\vec{r}, t))
$$

where $\delta \rho(\vec{r}, t)=\rho(\vec{r}, t)-\rho_{0}(t)$ is the density perturbation. $\rho_{0}(t)$ is the unperturbed density. In first order of perturbation theory, the gravitational potential does not depend on time when expressed in terms of comoving coordinates, i.e. $\phi(\vec{r}=a(t) \vec{x}, t)=\phi(\vec{x})$. Moreover $\delta(\vec{r}, t) \equiv \frac{\delta \rho(\vec{r}, t)}{\rho_{0}(t)}$ has the form $\delta(\vec{r}=a(t) \vec{x}, t)=a(t) \delta(\vec{x})$. Hence

$$
\vec{\tau}(t)=\rho_{0}(t) a(t)^{4} \int_{V} d^{3} x \delta(\vec{x}) \vec{x} \times\left(-\vec{\nabla}_{x} \phi(\vec{x})\right)
$$

Eq. (3.21) shows that the direction of the torque is time independent. Hence the rotation axis is time independent, as in Eq. (3.3). Furthermore, since $\rho_{0}(t) \propto a(t)^{-3}, \tau(t) \propto a(t) \propto t^{\frac{2}{3}}$ and hence the angular momentum increases with time proportionally to $t^{\frac{5}{3}}$. Since $R(t) \propto t^{\frac{2}{3}+\frac{2}{9 \epsilon}}$, tidal torque theory predicts the time dependence of Eq. (3.3) provided $\epsilon=0.33$. This value of $\epsilon$ is in the range, $0.25<\epsilon<0.35$, predicted by the evolved spectrum of density perturbations and supported by the evidence for caustic rings. So the time dependence in Eq. (3.3) is accounted for. 


\section{5. $j_{\max }$}

Here we compare the average value of $j_{\max }$ implied by the evidence for caustic rings with the amount of angular momentum expected from tidal torquing. The amount of angular momentum acquired by a galaxy through tidal torquing can be reliably estimated by numerical simulation because it does not depend on any small feature of the initial mass configuration, so that the resolution of present simulations is not an issue in this case. The amount of galactic angular momentum is usually given in terms of the dimensionless quantity [42]

$$
\lambda \equiv \frac{\mathcal{L}|\mathcal{E}|^{\frac{1}{2}}}{G \mathcal{M}^{\frac{5}{2}}},
$$

where $\mathcal{L}$ is the angular momentum of the galaxy, $\mathcal{M}$ its mass and $\mathcal{E}$ its net mechanical (kinetic plus gravitational potential) energy. $\lambda$ was found in numerical simulations to have a broad distribution with median value 0.05 [43]. $\lambda$ may also be estimated from observations of the luminous matter by making some assumptions, in particular the assumption that the angular momentum per unit mass of the disk and the halo are equal. Using such methods, Hernandez et al. [44] derived the $\lambda$ distribution of a large sample of spiral galaxies from the Sloan Digital Sky Survey and found it to be consistent with the expectations from numerical simulations.

On the other hand, in the caustic ring model, the dimensionless measure of galactic angular momentum is $j_{\max }$. The evidence for caustic rings implies that the $j_{\max }$ distribution is peaked at $j_{\max } \simeq 0.18$. In case of isotropic infall $(v=0)$, the relationship between $j_{\max }$ and $\lambda$ is [4]

$$
\lambda=\sqrt{\frac{6}{5-3 \epsilon}} \frac{8}{10+3 \epsilon} \frac{1}{\pi} j_{\max } .
$$

For $\epsilon=0.33$, Eq. (3.23) implies $\lambda / j_{\max }=0.283$. Hence there is excellent agreement between $j_{\max } \simeq 0.18$ and $\lambda \sim 0.05$ when $v=0$. That the agreement is so good is likely somewhat fortuitous since neither the $\lambda$ nor the $j_{\max }$ distribution is very well established. Also, because the extent of galactic halos is not uniformly agreed upon, there is some amibiguity in the definition of the quantities, $\mathcal{L}, \mathcal{E}$ and $\mathcal{M}$, that enter $\lambda$. In deriving Eq. (3.23), the halo was taken to extend all the way to the turnaround radius [4]. In obtaining the $\lambda$ distribution from numerical simulations, a definition of a galactic halo convenient in numerical simulations is used [43]. The two definitions are not clearly equivalent. All together the agreement between $\lambda$ and $j_{\text {max }}$ is significant only within some factor of order one, perhaps as large as 2 .

For $v \neq 0$, the relationship between $\lambda$ and $j_{\max }$ is more difficult to derive because of the lack of spherical symmetry. A calculation implies that the RHS of Eq. (3.23) is multiplied by a factor of order $\frac{1+v / 2}{1+v / 3}$. In a future publication, we will justify this factor and produce refinements to it [41]. If $v$ is much larger than one, the RHS of Eq. (3.23) is multiplied by $3 / 2$ so that $\frac{\lambda}{j_{\max }} \simeq 0.426$ for $\epsilon=0.33$. The values $\lambda \sim 0.05$ and $j_{\max } \simeq 0.18$ are then consistent at the $50 \%$ level only.

\section{B. Baryons and WIMPs}

The gravitational forces produced by the axion BEC act not only on the axions themselves but also on all other particles present. In particular, the axion BEC interacts gravitationally 
with baryons, and with WIMPs if WIMPs are present. The condition for baryons/WIMPs to acquire net overall rotation by gravitational interaction with the axion BEC is heuristically

$$
4 \pi G n m m^{\prime} \ell>m^{\prime} \dot{v}
$$

where $m^{\prime}$ is the baryon/WIMP mass. The accelerations $\dot{v}$ necessary to acquire net overall rotation are the same for baryons/WIMPs as for axions. Since $m^{\prime}$ cancels out of the inequality (3.24), conditions (3.24) and (3.2) are the same. It was found in ref. [5] that, if the dark matter is entirely axions, the inequality (3.2) is satisfied by a factor of order 30 . Therefore conditions (3.2) and (3.24) are equivalent to

$$
n m \gtrsim \frac{1}{30} \rho_{\mathrm{DM}}
$$

where $\rho_{\mathrm{DM}}$ is the total cold dark matter density. If the axion fraction of cold dark matter is larger than of order $3 \%$, we expect the axion BEC to acquire net overall rotation and to entrain the baryons and WIMPs along.

The baryons and WIMPs do not form a BEC but, by being in thermal contact with the axion BEC, they behave in very much the same way. Indeed, thermal contact between baryons/WIMPs and axions implies that they have the same temperature $T$ and the same angular velocity $\omega$, as was discussed in subsection II.A. The temperature of axions is certainly smaller than the typical kinetic energy of axions in a galactic halo because axions with larger kinetic energy would escape. Since the typical halo velocity is $v \sim 10^{-3} c$,

$$
T \lesssim \frac{1}{2} m v^{2} \simeq 6 \cdot 10^{-8} \mathrm{~K}\left(\frac{m}{10^{-5} \mathrm{eV}}\right)
$$

The velocity dispersion of the baryons and WIMPs, being much heavier than the axions but at the same temperature, is tiny: less than $30 \mathrm{~cm} / \mathrm{s} \mathrm{if} m^{\prime} \geq 1 \mathrm{GeV}$ and $m<10^{-3} \mathrm{eV}$. The temperature of baryons and WIMPs is thus effectively zero. Baryons and WIMPs are therefore in their own state of lowest available $\eta=\epsilon-\omega l$, with the same $\omega$ as the axions. That state may be derived by the same methods as we used for the axions in Section II.A. The outcome is that the baryons/WIMPs are in a state of rigid rotation (again not in the three dimensional sense, but in the sense that each spherical shell rotates rigidly with angular velocity proportional to $r^{-2}$ where $r$ is the shell's radius) with the same velocity field, Eq. (3.15), as the axion BEC. The underlying reason for this outcome is simple. If the baryons and WIMPs were not locally at rest with respect to the axion BEC, entropy could be generated by bringing them to such a state. Furthermore, as was the case for the axions, the lowest $\eta$ state is one where all the baryons and WIMPs are near the equator. We assume again, as we did for axions, that the baryons/WIMPs start to move towards the equator but that there is not enough time for them to all get there. Thus the baryon/WIMP infall rate is taken to have the same functional dependence on $t$ and $\theta$ as for axions

$$
\frac{d M^{\prime}}{d \Omega d t}=N_{v^{\prime}}(\sin \theta)^{v^{\prime}} \frac{M^{\prime}}{6 \pi \epsilon t}
$$

but we allow a different value $v^{\prime}$ for the $v$ parameter. Indeed we expect that the axions move to the equator first and that the baryons/WIMPs follow them there. Since the baryons/WIMPs are locally at rest with respect to the axion BEC, their angular momentum 
distribution on the turnaround sphere is the same as in Eq. (3.3) but with $m$ replaced by $m^{\prime}$ :

$$
\overrightarrow{l^{\prime}}(\hat{n}, t)=m^{\prime} j_{\max } \hat{n} \times(\hat{z} \times \hat{n}) \frac{R(t)^{2}}{t}
$$

Since the WIMPs fall in with the same initial velocity distribution as the axions, they move in the same way after falling onto the galaxy and produce the same caustic structures. The baryons also fall in the same way initially, but being collisionfull, separate from the axions and WIMPs after shell crossing starts.

\section{COMPARISON WITH OBSERVATIONS}

Here we discuss two observations that appear related to the physics described in Section III. The first is a measurement of the angular momentum distribution of baryonic matter in dwarf galaxies by van den Bosch et al. [12]. The second is the typical size of the observed rises in the Milky Way rotation curve, compared to the prediction from caustic rings of dark matter [1].

\section{A. Baryonic angular momentum distribution}

In the first part of this subsection we recount a discrepancy between the observed and predicted angular momentum distributions of baryons in galaxies if the dark matter is ordinary cold dark matter, such as WIMPs. In the second part we show how the discrepancy is resolved if the dark matter is axions.

\section{If the dark matter is all WIMPs}

Since we assume in this subsection that none of the dark matter is axions, the considerations of Section III do not apply.

By the principle of equivalence, tidal torquing gives the same amount and the same distribution of specific angular momentum (i.e. angular momentum per unit mass) to baryons and to dark matter before they fall onto galactic halos. Let us assume, to start with, that the individual angular momentum of each particle is conserved from its turnaround till today. In that case the observed amount and distribution of baryonic specific angular momentum is the same as predicted for dark matter by numerical simulations. It was mentioned already in subsection III.A.5 that the amount of specific angular momentum observed in the baryonic components of disk galaxies is consistent with the amount expected from numerical simulations, lending support to the hypothesis that the angular momentum of each particle is conserved. However, the observed specific angular momentum distribution of baryons in disk galaxies differs markedly from that predicted by numerical simulations for WIMP dark matter. The predicted distribution has many more particles with low specific angular momentum than the observed distribution and a compensating (to keep the average the same) population of particles with much higher specific angular momentum. The simulations predict too high a concentration of baryons at the centers of galaxies.

At first it may appear that the solution to this discrepancy is simply to abandon the notion that the angular momentum of individual particles is conserved after they have fallen 
onto the galaxy. However, when the processes that allow angular momentum exchange are modeled, it is found that they aggravate the discrepancy rather than resolve it. Frictional forces among baryons have the general effect of removing angular momentum from baryons that have little angular momentum and transferring it to baryons that have a lot. Dynamical friction of dark matter on clumps of baryonic matter has the general effect of tranferring angular momentum from the baryons to the dark matter. Both processes tend to concentrate baryons at galactic centers even more, aggravating the discrepancy [13]. The discrepancy is commonly referred to as the 'galactic angular momentum problem'. See ref. [14] for a review.

The problem is thrown into sharp relief by comparing the universal angular momentum distribution obtained by Bullock et al. from numerical simulations [45] with the observed angular momentum distribution of baryons in dwarf galaxies [12]. Bullock et al. found that the specific angular momentum distributions of the galaxies in simulations are all well fitted by a single two parameter function:

$$
\frac{d M}{d l}=\frac{\mu M_{v} l_{0}}{\left(l_{0}+l\right)^{2}} \quad \text { for } \quad 0 \leq l \leq l_{\max }=\frac{l_{0}}{\mu-1}
$$

where $\mu>1$, and $M_{v}$ is the halo's virial mass. Each galaxy has its own value of $\mu$ and $l_{\max }$. The distribution of $\log _{10}(\mu-1)$ values for the galaxies in the simulations is nearly Gaussian with average -0.6 and standard deviation 0.4 , implying that $90 \%$ of halos have $0.06<\mu-1<$ 1.0. The median $\mu$ value is 1.25 . The ratio of the average specific angular momentum $l_{\mathrm{av}}$ to the maximum specific angular momentum is given in terms of the parameter $\mu$ by

$$
\frac{l_{\mathrm{av}}}{l_{\max }}=(\mu-1)\left[\mu \ln \left(\frac{\mu}{\mu-1}\right)-1\right] .
$$

The broad distribution of $\mu$ values implies a correspondingly broad distribution of $\frac{l_{\mathrm{av}}}{l_{\max }}$ values with average near $0.25[12]$.

van den Bosch et al. [12] derived the baryonic angular momentum distribution of fourteen dwarf galaxies from observations by Swaters [46]. The distributions are shown in Fig. I which is a reproduction of the relevant figure in ref. [12]. The prediction of Eq. (4.1) with $\mu=1.25$, the median value, is shown as a solid line in each panel. The observed distributions are markedly different from the prediction of Eq. (4.1). Perhaps the most striking difference is that Eq. (4.1) predicts $\frac{d M}{d l}$ to be maximum at $l=0$ whereas the observed distributions appear to go to zero at $l=0$ and have their maxima around $l=l_{\text {av }}$. Another striking difference, pointed out in ref. [12], is that the observed values of $\frac{l_{\mathrm{av}}}{l_{\max }}$ are strongly peaked near 0.375 . This is apparent from the fact that many of the distributions in Fig. I end at $l_{\max } \simeq 2.6 l_{\mathrm{av}}$. As mentioned, the numerical simulations predict $\frac{l_{\mathrm{av}}}{l_{\max }}$ to have a broad distribution with median value 0.25 . If this were so, the distributions in Fig. I would end at a wide variety of $\frac{l_{\max }}{l_{\text {av }}}$ values and half of these values would be larger than 4 .

\section{If the dark matter is axions, at least in part}

As described in Section III, the angular momentum distributions of the baryons and WIMPs are modified by gravitational interactions with the axion BEC. The outcome is Eq. (3.28) for the angular momentum distribution on the turnaround sphere and Eq. (3.27) for the infall rate. We assume that the angular momentum of each particle is conserved after 
it crosses the turnaround sphere. Eq. (3.28) implies for the angular momentum distribution on the turnaround sphere at time $t$

$$
l(\theta, t)=\hat{z} \cdot \vec{l}(\vec{n}, t)=l_{\max }(\sin \theta)^{2}\left(\frac{t}{t_{0}}\right)^{\frac{5}{3}}
$$

where $t_{0}$ is the present age of the universe and

$$
l_{\max }=m j_{\max } \frac{R_{0}^{2}}{t_{0}}
$$

is the angular momentum of particles falling in along the galactic plane today. $R_{0}$ is the present turnaround radius. We are removing the primes from $\overrightarrow{l^{\prime}}, m^{\prime}, M^{\prime}$ and so forth to avoid cluttering the equations unnecessarily. To obtain Eq. (4.3) from Eq. (3.28) we used the fact that $l \propto t^{\frac{5}{3}}$; see subsection III.A.4. The angular momentum distribution today is

$$
\frac{d M}{d l}(l)=\int d \Omega \int_{0}^{t_{0}} d t \frac{d M}{d \Omega d t}(\theta, t) \delta(l-l(\theta, t))
$$

Substituting Eqs. (3.27) and (4.3) and carrying out the $t$ integration, one finds for $\epsilon=1 / 3$

$$
\frac{d M}{d l}(l)=\frac{6}{5} \frac{M_{0}}{l_{\max }}\left(\frac{l}{l_{\max }}\right)^{\frac{1}{5}} I_{v}\left(\frac{l}{l_{\max }}\right)
$$

where

$$
I_{v}(r)=N_{v} \int_{0}^{\sqrt{1-r}} \frac{d x}{\left(1-x^{2}\right)^{\frac{6}{5}-\frac{v}{2}}}
$$

The predicted angular momentum distributions are shown in Fig. II for $v=0,0.5,1.0,1.5$, $2.0,3.0,5.0,10 ., 50$. and 100. For $v=0, \frac{d M}{d l}(l)$ has a sharp cusp at $l=0$ with $\frac{d M}{d l}(0)=3 \frac{M_{0}}{l_{\max }}$. However, as soon as $v>0, \frac{d M}{d l} \propto l^{\frac{1}{5}}$ near $l=0$. For $v$ in the range 0.5 to $2.0, \frac{d M}{d l}(l)$ is qualitatively similar to the angular momentum distributions found by van den Bosch et al. in dwarf galaxies.

The average angular momentum is

$$
l_{\mathrm{av}}=\frac{1}{M_{0}} \int_{0}^{l_{\max }} d l l \frac{d M}{d l}(l)=l_{\max } \frac{3}{11} \frac{\sqrt{\pi}}{2^{v}}\left(\frac{v}{2}+1\right) \frac{\Gamma(v+2)}{\Gamma\left(\frac{v}{2}+1\right) \Gamma\left(\frac{v}{2}+\frac{5}{2}\right)} .
$$

$l_{\max } / l_{\text {av }}$, plotted in Fig. III, decreases monotonically with $v$, from 2.75 at $v=0$ to 1.83 at $v=\infty$. In the range $0.5<v<2.0, l_{\max } / l_{\text {av }}$ ranges from 2.56 to 2.29 . So we find that in the axion case 1) the $l_{\max } / l_{\text {av }}$ distribution is sharply peaked, like the observed distribution, and 2 ) it is peaked at roughly the same value (2.6) as the observed distribution.

\section{B. Enhanced caustic rings}

The observational evidence in support of the caustic ring halo model is summarized in ref. [9]. A large part of that evidence is based on the existence of statistically significant correlations between bumps in galactic rotation curves, consistent with the assumption that 
some of the bumps are caused by caustic rings of dark matter and that the caustic ring radii obey Eq. (3.4) [11, 47]. Additional evidence is provided by the fact that the bumps in the high resolution inner rotation curve of the Milky Way published in ref. [48] are kinky, i.e. they start with an upward kink and end with a downward kink [11]. The kinks are explained by the fact that the dark matter density diverges at caustic surfaces [8]. Yet more evidence is provided by the existence of a triangular shape in the IRAS (Infrared Astronomical Sattelite) map of the galactic plane in one of the two tangent directions to the nearest caustic ring $(n$ $=5)$. The position of the triangular shape coincides in galactic longitude with the position of the rise in the rotation curve associated with that caustic ring. The triangular shape is explained as the imprint of the gravitational field of the caustic ring on dust and gas in the galactic disk.

There is however a puzzle with the interpretation of the evidence: the effects attributed to caustic rings are too large compared to theoretical expectation. Specifically, the bumps in the Milky Way rotation curve are on average a factor 5 larger [11] than expected in the caustic ring model if the infall is isotropic [7-9] and if the bumps are due solely to the caustic rings themselves. The sizes of the bumps are not actually predicted precisely by the caustic ring model. They are given as a product of two factors, one of which is predicted by the model. The other factor depends on details that the model (in its present state) does not predict and which fluctuate from one caustic ring to the next. Nonetheless, this second factor is expected to be generally of order one. There is no reason why its average should be five. See refs. [8, 11] for details.

To account for this discrepancy, ref. [11] proposed that the rises in rotation curves are amplified by gas that is gravitationally attracted to, and accreted onto, the caustic rings. The square of the velocity dispersion of gas in the galactic disk is sufficiently small compared to the gravitational potential ripples caused by caustic rings that a large amplification factor is plausible. However, in this proposal it is hard to understand the kinkiness of the bumps in the Milky Way rotation curve since the gas distribution would follow the gravitational potential of the caustic rings, which is much smoother than the density of the caustic rings.

The physics discussed in Section III suggests a simpler and more compelling explanation, to wit that the infalling axion BEC has a big vortex along the galactic symmetry axis and hence that the infall is not isotropic. The caustic rings are enhanced because more dark matter falls in near the galactic plane. If the infall rate is given by Eq. (3.18), the density of the flow producing the caustic ring is increased by the factor $N_{v}$ given in Eq. (3.19). For $N_{v}$ to be of order five, $v$ must be of order forty.

There is a restriction on how large $v$ can be because a caustic ring is partly erased if the infalling dark matter is too concentrated near the galactic plane. Using the description in ref. [8], one readily finds that caustic rings are formed in the flow of particles whose declination $\alpha \equiv \frac{\pi}{2}-\theta$ at their last turnaround is less in magnitude than $\alpha_{m}=\frac{1}{2} \sqrt{\frac{u}{s}} \tau_{0} \simeq \sqrt{\frac{p}{2 a}}$ where $p$ is the width of the caustic ring. See ref. [8] for definitions of $u, s, \tau_{0}$. Some of the caustics may be partly erased but not $n=5$ since, as mentioned above, its full cross-section appears in IRAS maps of the galactic plane; see http://www.phys.ufl.edu/ sikivie/triangle/index.htm. For that ring, $p=0.018 a$ and hence $\alpha_{m}=5.5^{\circ}$. Requiring $\left(\cos \alpha_{m}\right)^{v}>0.5$ allows $v$ as large as 150 and hence an enhancement by the factor $N_{v}$ as large as 10 . The rise in the Milky Way rotation curve associated with the $n=5 \mathrm{ring}$ is a factor three larger [11] than expected in the isotropic model $(v=0)$, and therefore consistent with the appearance of the full cross-section of the caustic ring in the aforementioned IRAS map.

If the existence of a big vortex is the correct explanation for the enhanced effect of caustic 
rings of dark matter on galactic rotation curves, we may derive a lower limit on the fraction $X_{a}$ of dark matter that is axions. Let $X_{W}=1-X_{a}$ be the dark matter fraction in WIMPs or some other form of ordinary cold dark matter. We need

$$
X_{a} N_{v}+X_{W} N_{v^{\prime}} \simeq 5
$$

to account for the average strength of the rises in the Milky Way rotation curve. On the other hand $N_{v} \lesssim 10$, otherwise the corners of the triangular feature in the IRAS map get erased. Also $v^{\prime} \lesssim 5$, otherwise the angular momentum distribution of baryonic matter becomes too dissimilar to the distributions observed by van den Bosch et al.; compare Figs. II and III. Eq. (3.19) implies then $N_{v^{\prime}} \lesssim 2$. Combining all this, one obtains

$$
X_{a} \gtrsim \frac{3}{8},
$$

i.e. a lower limit of approximately $37.5 \%$ on the axion dark matter fraction.

\section{SUMMARY}

The goal of this paper was to increase our understanding of the behaviour of axion BEC dark matter before it falls into the gravitational potential well of a galaxy. In particular we wanted to see how axion BEC vortices appear and evolve, and whether they have implications for observation.

In Section II, we discussed the properties of rotating many body systems in thermal equilibrium. We showed that the widely used self-gravitating isothermal sphere model is an unacceptably poor description of galactic halos as soon as angular momentum is introduced. We showed that the vortices that appear in a BEC of quasi-collisionless particles, such as an axion BEC, attract each other, in contrast to the repulsive behavior of the vortices in superfluid ${ }^{4} \mathrm{He}$ and dilute gases. We showed that vortices in any BEC appear only as part of the process of rethermalization after the BEC has been given angular momentum. Neither the thermalization of a BEC nor the appearance of its vortices is described by the GrossPitaevskii equation. That equation describes the behavior of the BEC, including the motion of its vortices, only after it has formed.

In Section III, we used the results of Section II to try and predict the behavior of axion BEC dark matter before it falls into the gravitational potential well of a galaxy. As angular momentum is acquired by the axion BEC through tidal torquing, the axions go to a state where all spherical shells rotate rigidly about a common axis, with angular velocity proportional to $r^{-2}$ where $r$ is the radius of the shell. The resulting angular momentum distribution on the turnaround sphere, Eq. (3.3), is precisely and in all respects that which accounts for the evidence for caustic rings of dark matter. Because axion BEC vortices are attractive, we expect that most join into one big vortex. The radius of this big vortex is smaller than but of order the radius of the first caustic ring made by the axion BEC as it falls in and out of the galaxy. We modified the caustic ring model of galactic halos to include the presence of the big vortex. Whereas the previous version of that model assumed that the infall is isotropic, the new version assumes that the infall rate is given by Eq. (3.18) where $v$ parametrizes the size of the big vortex.

The rate at which baryons and WIMPs reach thermal equilibrium with the axion BEC was found to be qualitatively the same as the rate at which axions reach thermal equilibrium 
among themselves. That thermalization rate is larger than the Hubble rate provided the axion dark matter fraction is more than of order 3\%. Because baryons and WIMPs are much heavier than axions, the temperature of baryons and WIMPs is effectively zero when they are in thermal contact with the axions. In that case, baryons and WIMPs acquire the same velocity distribution as the axion BEC before falling onto galactic halos, and WIMPs produce the same caustic rings as axions do, and at the same locations. We expect the baryons and WIMPs to produce their own big vortex although with radius smaller than the radius of the big vortex in axions. The specific angular momentum distribution of baryons and WIMPs on the turnaround sphere is the same as for axions. The infall rate is also the same but with a smaller value $v^{\prime}$ of the parameter $v$.

In Section V, we compared the specific angular momentum distribution predicted for baryons, when the axion dark matter fraction is more than of order $3 \%$, with the specific angular momentum distribution of baryons observed in dwarf galaxies. They are qualitatively similar for $0.5 \lesssim v^{\prime} \lesssim 2$. Moreover, in this range the ratio $l_{\max } / l_{\mathrm{av}}$ of maximum to average specific angular momentum is predicted to be near 2.4. This is in qualitative agreement with the observed distributions since most of the latter have $l_{\max } / l_{\mathrm{av}} \simeq 2.6$. In contrast, if the dark matter is all WIMPs, the specific angular momentum distribution differs markedly from the observed distributions; see Fig. I. Furthermore, $l_{\max } / l_{\text {av }}$ is predicted in the WIMP case to vary from galaxy to galaxy, the median value being 4 and the $90 \%$ range from 2.6 to 8.1. The ability of axion dark matter to qualitatively explain the observed angular momentum of baryons in dwarf galaxies is further evidence that at least part of the dark matter is axions.

The appearance of a large vortex in the axion BEC provides a plausible solution to a past puzzle, namely that the rises in galactic rotation curves attributed to caustic rings of dark matter are typically a factor 5 larger than expected when the dark matter infall is assumed to be isotropic. The presence of a big vortex implies that more dark matter falls in along the galactic plane and hence that the density of the flows producing the caustic rings is increased. If all the dark matter is axions, the factor 5 enhancement is accounted for if $v$ is of order 40. If the dark matter is partly axions and partly WIMPs, with the axion fraction more than of order 3\%, axions and WIMPs co-produce the caustic rings. If one requires $v^{\prime}<5$ to have an acceptable fit between the predicted and observed specific angular momentum distribution of baryons in dwarf galaxies, the caustic ring enhancement of a factor of five can only be accounted for if at least $37 \%$ of the dark matter is axions.

\section{Acknowledgments}

We thank Leslie Rosenberg, Tom Quinn, Tanja Rindler-Daller, Paul Shapiro, Frank van den Bosch and Mark Srednicki for stimulating discussions. This work was supported in part by the U.S. Department of Energy under grant DE-FG02-97ER41209 at the University of Florida, the National Science Foundation under Grant No. PHYS-1066293 at the Aspen Center for Physics, and the National Science Foundation under grant PHY11-25915 at the Kavli Institute for Theoretical Physics at UC Santa Barbara. 
[1] Reviews include: Particle Dark Matter edited by Gianfranco Bertone, Cambridge University Press 2010; E.W. Kolb and M. Turner, The Early Universe, Addison Wesley 1990.

[2] P.A.R. Ade et al. (the Planck Collaboration), arXiv:1303.5076.

[3] P. Sikivie and Q. Yang, Phys. Rev. Lett. 103 (2009) 111301.

[4] P. Sikivie, Phys. Lett. B 695 (2011) 22.

[5] O. Erken, P. Sikivie, H. Tam and Q. Yang, Phys. Rev. D85 (2012) 063520.

[6] A. Natarajan and P. Sikivie, Phys. Rev. D73 (2006) 023510.

[7] P. Sikivie, Phys. Lett. B432 (1998) 139.

[8] P. Sikivie, Phys. Rev. D60 (1999) 063501.

[9] L. Duffy and P. Sikivie, Phys. Rev. D78 (2008) 063508.

[10] P. Sikivie, I. Tkachev and Y. Wang, Phys. Rev. Lett. 75 (1995) 2911; Phys. Rev. D56 (1997) 1863.

[11] P. Sikivie, Phys. Lett. B567 (2003) 1.

[12] F.C. van den Bosch, A. Burkert and R.A. Swaters, MNRAS 326 (2001) 1205.

[13] J.F. Navarro and M. Steinmetz, Ap. J. 528 (2000) 607, and references therein.

[14] A.M. Burkert and E. D'Onghia, ASSL 319 (2004) 341.

[15] S.-J. Sin, Phys. Rev. D50 (1994) 3650; J. Goodman, New Astronomy Reviews 5 (2000) 103; W. Hu, R. Barkana and A. Gruzinov, Phys. Rev. Lett. 85 (2000) 1158; E.W. Mielke and J.A. Vélez Pérez, Phys. Lett. B671 (2009) 174; J.-W. Lee and S. Lim, JCAP 1001 (2010) 007; A. Lundgren, M. Bondarescu, R. Bondarescu and J. Balakrishna, Ap. J. 715 (2010) L35; D.J. Marsh and P.G. Ferreira, Phys. Rev. D82 (2010) 103528; V. Lora et al., JCAP 02 (2012) 011.

[16] T. Rindler-Daller and P. Shapiro, MNRAS 422 (2012) 135, and references therein.

[17] S. Chandrasekhar, An Introduction to the Theory of Stellar Structure, U. of Chicago Press, 1939.

[18] K. Huang, Statistical Mechanics, Wiley 1987.

[19] R.K. Pathria, Statistical Mechanics, Elsevier 1996.

[20] J.R. Ipser and P. Sikivie, Phys. Rev. D35 (1987) 3695.

[21] J. Binney and S. Tremaine, Galactic Dynamics, Princeton U. Press, 1987.

[22] P.F. Smith and J.D. Lewin, Phys. Rep. 187 (1990) 203.

[23] G. Jungman, M. Kamionkowski and K. Griest, Phys. Rep. 267 (1996) 195.

[24] P. Sikivie and J. Ipser, Phys. Lett. B291 (1992) 288.

[25] A. Natarajan and P. Sikivie, Phys. Rev. D72 (2005) 083513.

[26] K. Dolag, A.D. Dolgov and I.I. Tkachev, arXiv: 1210.8009.

[27] D. Lynden-Bell and R. Wood, MNRAS 138 (1968) 495.

[28] P.H. Chavanis, Astron. and Astrophys. 396 (2002) 315.

[29] C.J. Pethik and H. Smith, Bose-Einstein Condensation in Dilute Gases, Cambridge University Press 2002.

[30] L. Onsager, Nuovo Cimento 6 (1949) Suppl. 2, 249.

[31] R.P. Feynman, in Progress in Low Temperature Physics, Vol. 1, ed. C.J. Gorter, NorthHolland, Amsterdam 1955, Chapter 2.

[32] M. Abramowitz and I.A. Stegun, Handbook of Mathematical Functions, Dover Publications, 1965.

[33] L.M. Widrow and N. Kaiser, Astrophys. J. 416 (1993) L71. 
[34] V.K. Tkachenko, J. Exp. Theor. Phys. 22 (1966) 1282.

[35] E.J. Yarmchuk, M.J.V. Gordon and R.E. Packard, Phys. Rev. Lett. 43 (1979) 214.

[36] J.R. Abo-Shaeer, C. Raman, J.M. Vogels and W. Ketterle, Science 292 (2001) 476, and references therein.

[37] K. Saikawa and M. Yamaguchi, Phys. Rev. D87 (2013) 085010.

[38] G. Stromberg, Ap. J. 79 (1934) 460; F. Hoyle, in Problems of Cosmical Aerodynamics, ed. by J.M. Burgers and H.C. van de Hulst, 1949, p195, Dayton, Ohio: Central Air Documents Office; P.J.E. Peebles, Ap. J. 155 (1969) 393, and Astron. Ap. 11 (1971) 377.

[39] J.A. Fillmore and P. Goldreich, Ap. J. 281 (1984) 1; E. Bertschinger, Ap. J. Suppl. 58 (1985) 39.

[40] A.G. Doroshkevitch, Astrophysics 6 (1970) 320; P.J.E. Peebles, Ap. J. 277 (1984) 470; Y. Hoffman and J. Shaham, Ap. J. 297 (1985) 16.

[41] N. Banik and P. Sikivie, in preparation.

[42] P.J.E. Peebles, Ap. J. 155 (1969) 393.

[43] G. Efstathiou and B.J.T. Jones, MNRAS 186 (1979) 133; J. Barnes and G. Efstathiou, Ap. J. 319 (1987) 575;

[44] X. Hernandez, C. Park, B. Cervantes-Sodi and Y.-Y. Choi, MNRAS 375 (2007) 163.

[45] J.S. Bullock et al., Ap. J. 555 (2001) 240.

[46] R.A. Swaters, Ph.D. thesis, 1999, U. of Groningen, the Netherlands.

[47] W.H. Kinney and P. Sikivie, Phys. Rev. D61 (2000) 087305.

[48] D.P. Clemens, Ap. J. 295 (1985) 422. 


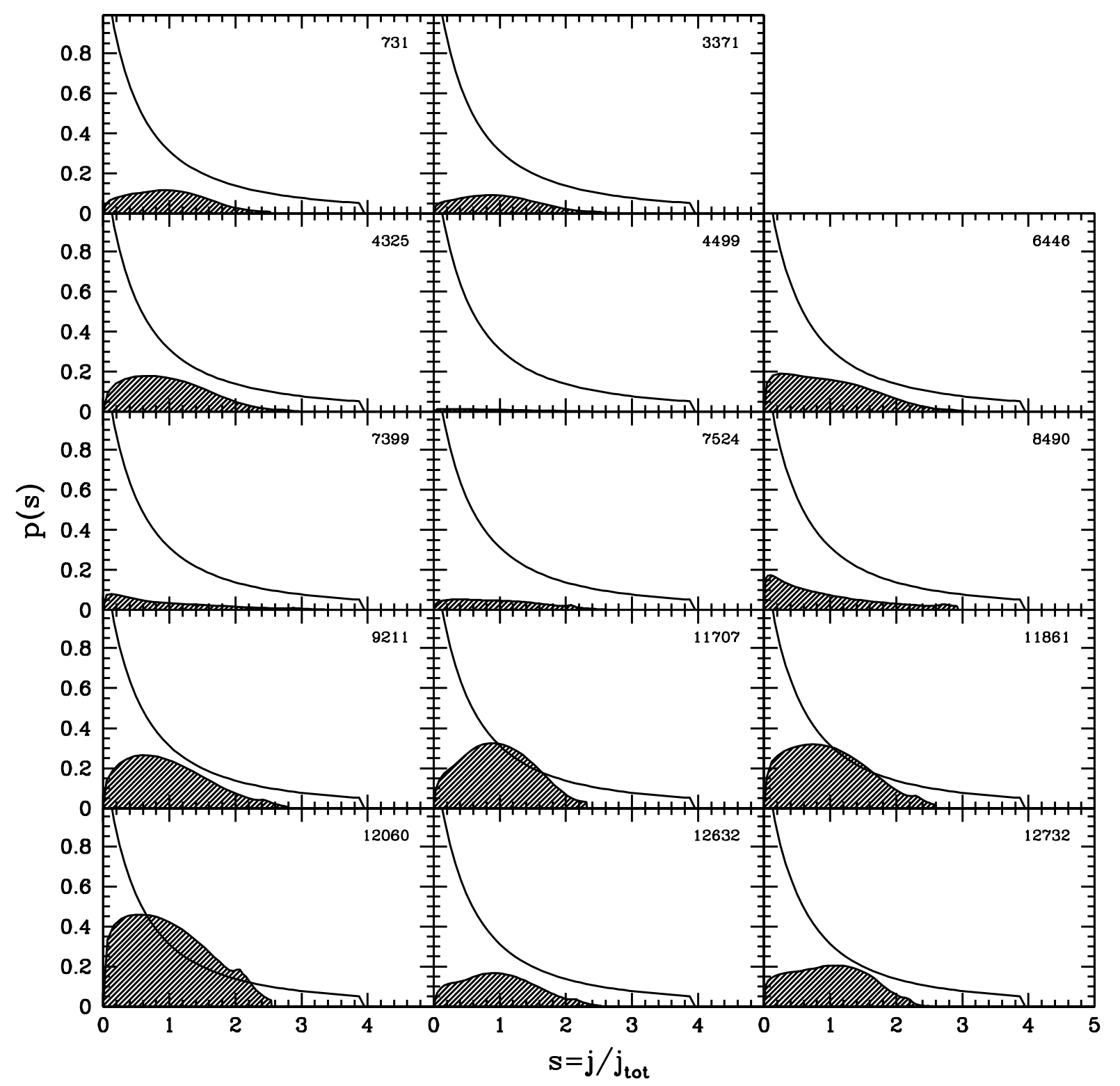

FIG. 1: Reproduction of Fig. 4 in the article The angular momentum content of dwarf galaxies: new challenges for the theory of galaxy formation by F.C. van den Bosch et al. [12]. The shaded areas indicate the specific angular momentum distributions of baryons in fourteen dwarf galaxies. In terms of the quantities defined in the text, $s=\frac{l}{l_{\mathrm{av}}}$ and $p(s) \propto \frac{d M}{d l}(l)$. The solid curve is the distribution, Eq. (4.1), predicted by numerical simulations of galaxy formation with ordinary cold dark matter, for $\mu=1.25$. 

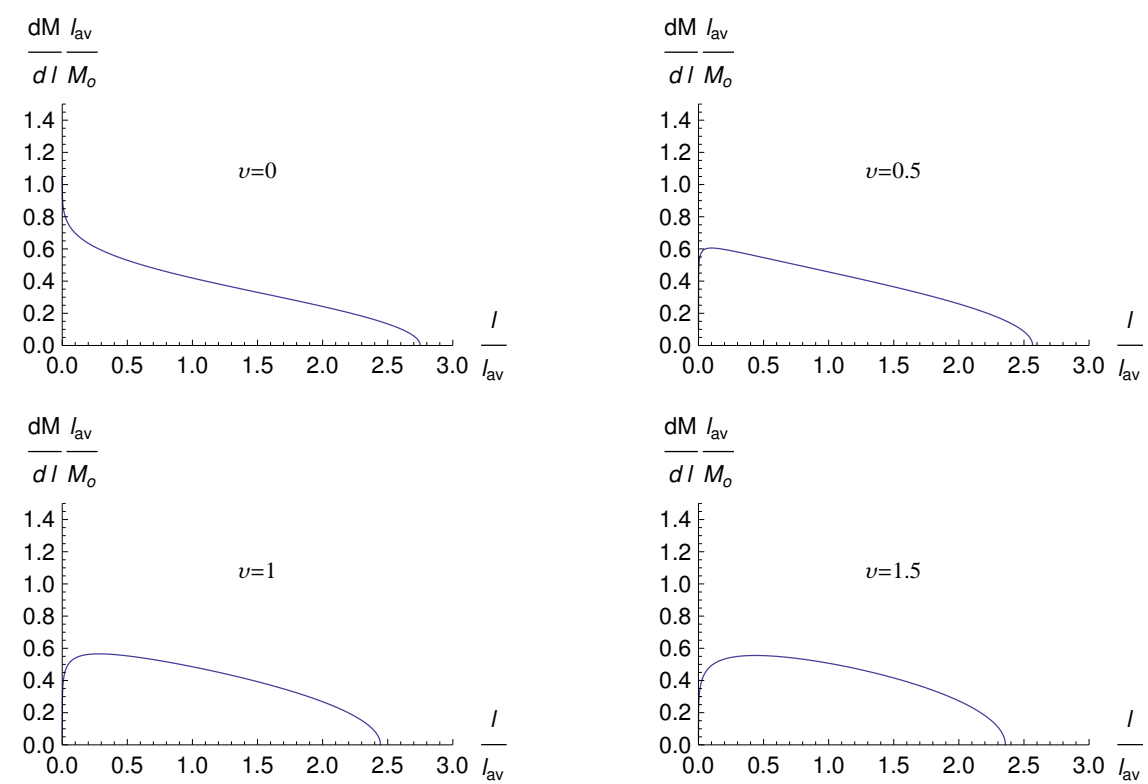

$\frac{\mathrm{dM}}{d l} \frac{l_{\mathrm{av}}}{M_{0}}$
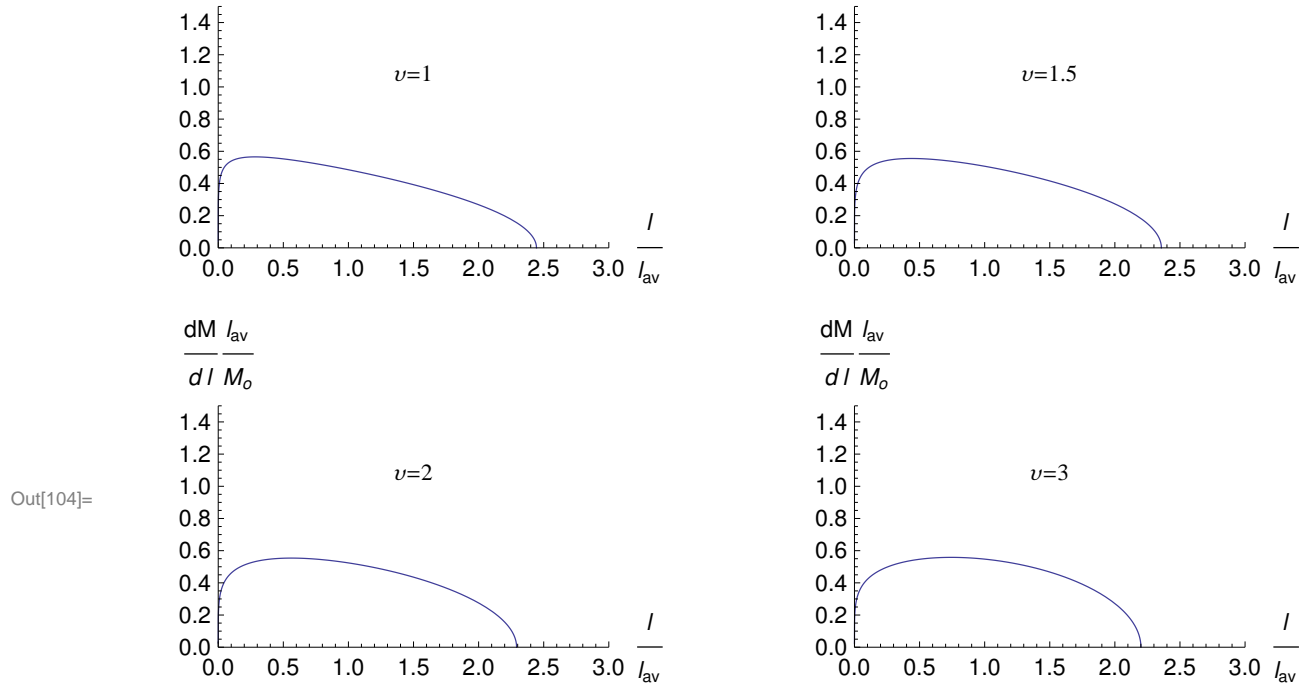

$\frac{\mathrm{dM}}{d l} \frac{I_{\mathrm{av}}}{M_{\mathrm{o}}}$
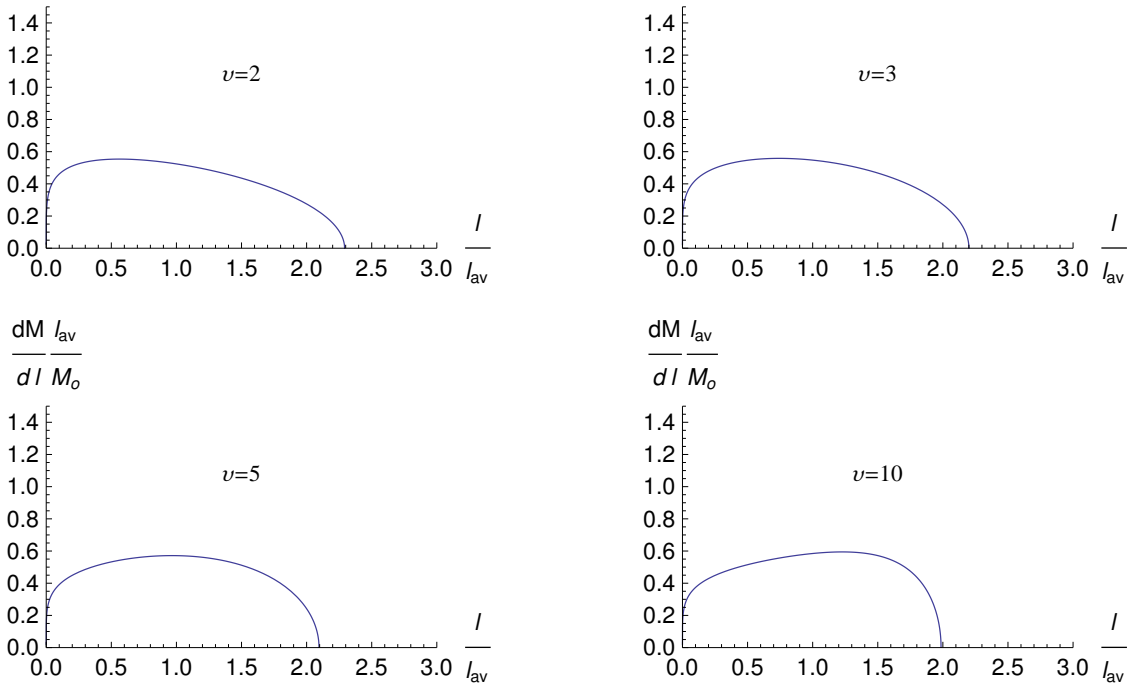

$\frac{\mathrm{dM}}{d l} \frac{l_{\mathrm{av}}}{M_{0}}$
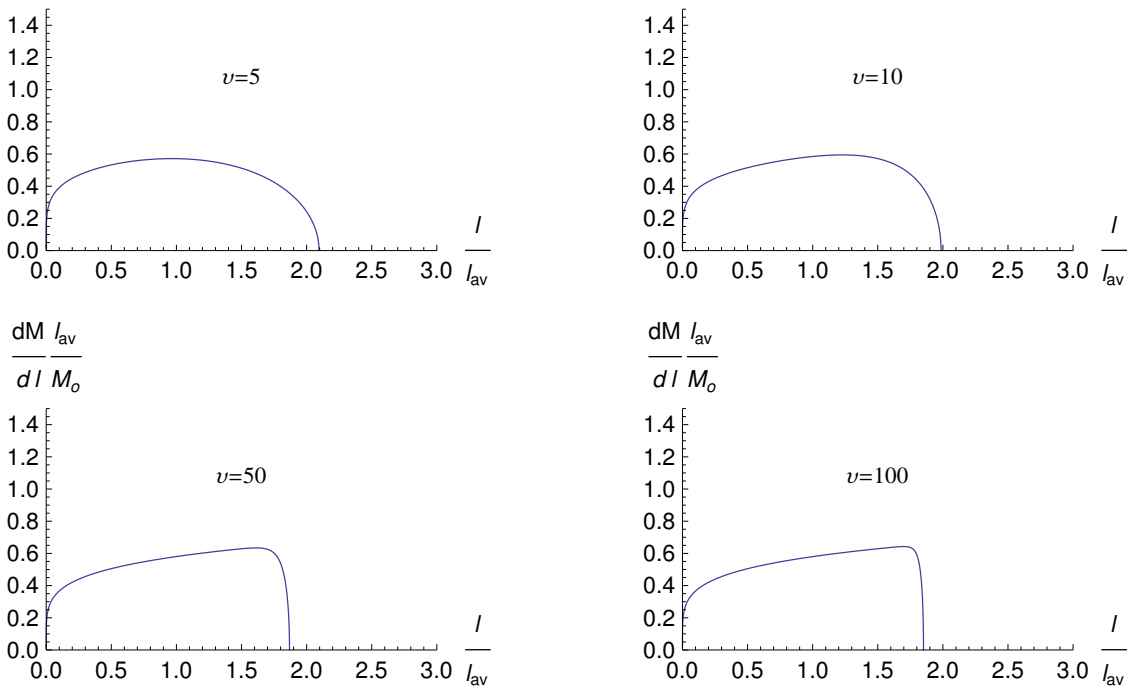

FIG. 2: Specific angular momentum distributions if the dark matter is axions, for various values of the parameter $v$. 


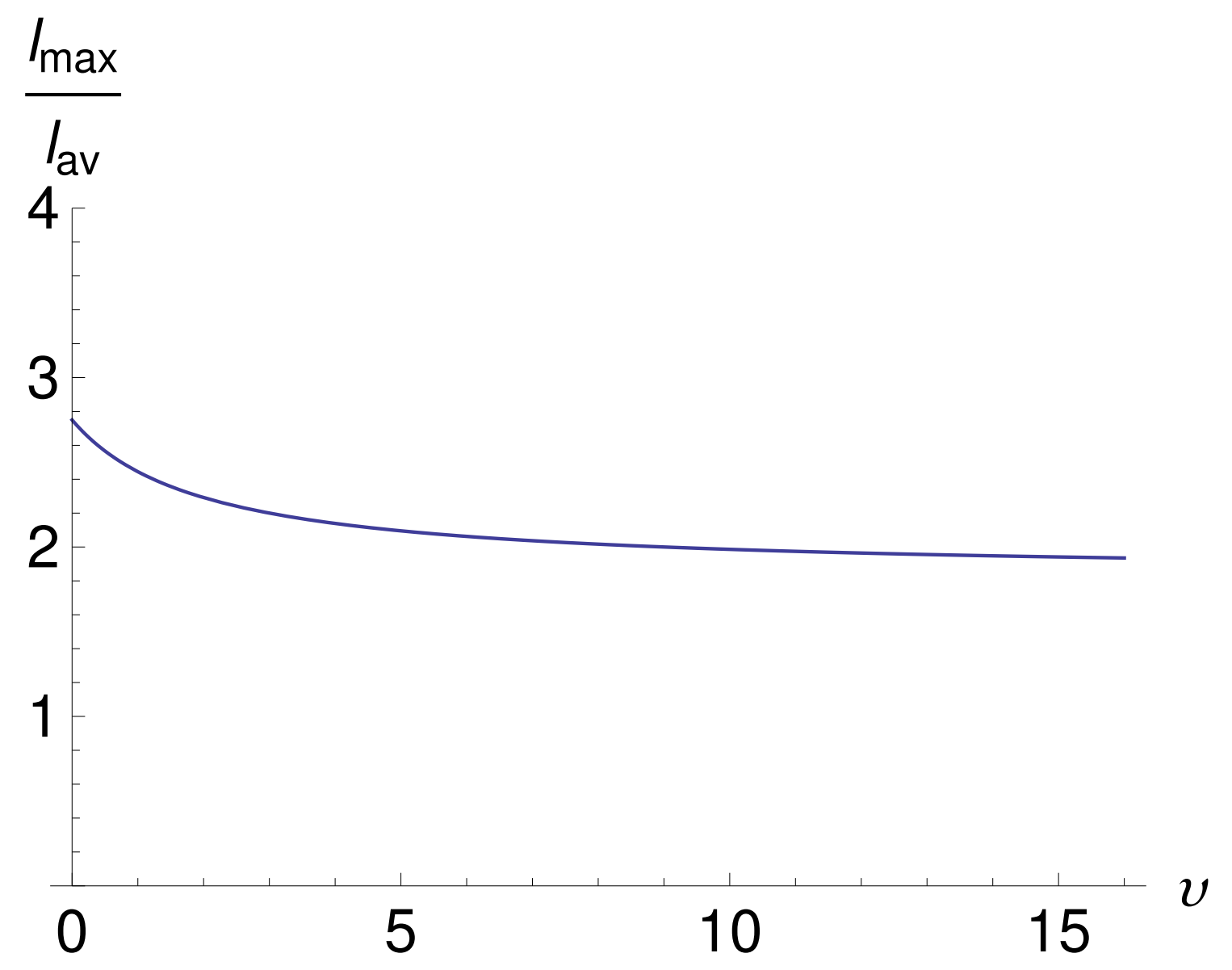

FIG. 3: The ratio of maximum to average angular momentum if the dark matter is axions, as a function of the parameter $v$. For the fourteen dwarf galaxies observed by van den Bosch et al. this ratio is narrowly peaked near 2.6. In numerical simulations of galaxy formation with ordinary cold dark matter, this ratio is predicted to vary from galaxy to galaxy over the range 2.6 to 8.1 (90 $\% \mathrm{CL})$, with median value 4.0 